\title{
Mass-Flux Characteristics of Reactive Scalars in the Convective Boundary Layer
}

\author{
Arthur C. Petersen, Cees Beets, Han van Dop, and Peter G. Duynkerke \\ Institute for Marine and Atmospheric Research Utrecht, Utrecht University, Utrecht, the Netherlands
}

A. Pier Siebesma

Royal Netherlands Meteorological Institute, De Bilt, the Netherlands

(Manuscript received 20 June 1997, in final form 13 March 1998)

\begin{abstract}
The transport of nonreactive and reactive bottom-up and top-down diffusing scalars in a solid-lid convective boundary layer is studied using large-eddy simulation (LES). The chemistry considered consists of an irreversible, binary reaction involving the bottom-up and top-down diffusing scalars. The mass-flux or top-hat characteristics of the reactive flow are determined. Also, several mass-flux schemes are run in an off-line mode, that is, with prescribed profiles of the mass flux and the updraft area fraction, and are compared to the LES. Top-hat approximations are found to capture about $25 \%$ of the covariance between two arbitrary (nonreacting or reacting) scalars and about $65 \%$ of the flux. Subplume fluxes are located either in the updraft for bottom-up diffusing scalars or in the downdraft for top-down diffusing scalars. The mass-flux scheme that is nearly identical to the exact plume-budget equations gives the best performance. For the parameterization of lateral exchange this mass-flux scheme includes gross exchange across the interface between updrafts and downdrafts, that is, includes also subinterface-scale exchange processes (like the other dynamical quantities also prescribed in an off-line mode using LES data). A simpler mass-flux scheme, which does not include the more sophisticated parameterizations of subplume fluxes and subinterface-scale lateral exchange, is found to perform only slightly worse. The results of this paper are also valid for the surface layer and lower mixed layer of the entraining convective boundary layer but not for the entrainment zone.
\end{abstract}

\section{Introduction}

For many years mass-flux schemes have been applied succesfully in the parameterization of scalar transport by cumulus convection (e.g., Arakawa and Schubert 1974; Tiedtke 1989). More recently, mass-flux schemes have been proposed as attractive candidates for the parameterization of transport in convective boundary layers (CBLs) - both the dry CBL and the stratocumulustopped CBL-for instance by Chatfield and Brost (1987) and Randall et al. (1992). The main difference between mass-flux schemes for cumulus and for the CBL is the decomposition that is applied: cloud-environment for cumulus and updraft-downdraft for the CBL. Another difference is that mass-flux formulas for the flux in cumulus resolve $80 \%-90 \%$ of the total flux (except near cloud base), as shown by Siebesma and Cuijpers (1995) for shallow cumulus, whereas mass-flux formulas for the flux in CBLs resolve about $65 \%$ of the total flux (Young 1988a; Schumann and Moeng 1991a; Wyngaard and Moeng 1992; de Laat and Duynkerke

Corresponding author address: A. C. Petersen, IMAU, Utrecht University, P.O. Box 80005, 3508 TA Utrecht, the Netherlands. E-mail: petersen@phys.uu.nl
1998). This difference is related to the presence of a potential barrier in cumulus convection. As a consequence, cloud updrafts occur in bursts in an otherwise quiet cloud layer and the vertical velocity distribution is highly positively skewed. Mass-flux schemes for the CBL are sensitive to the parameterization of the parts of the total flux that are not resolved by mass-flux formulas. These unresolved parts are called "subplume fluxes."

In this paper we study the scalar transport characteristics of different mass-flux schemes for transport of nonreactive and reactive scalars in the CBL. This study is part of the development of a new boundary layer parameterization for large-scale atmospheric chemistrytransport models (with horizontal grid sizes ranging from 50 to $500 \mathrm{~km}$ ) that includes turbulence-chemistry interactions (discussed below). The mass-flux schemes proposed earlier in the literature for transport of scalars, both in cumulus clouds and in CBLs, are combined, categorized, and evaluated using statistics from largeeddy simulation (LES) of a solid-lid CBL. The scalars studied are bottom-up and top-down diffusing scalars, either nonreacting or quickly reacting away. We run the mass-flux schemes in an off-line mode, that is, we prescribe the boundary layer height and the vertical profiles of the mass flux and the updraft area fraction (both 
determined from LES). Furthermore, we prescribe identical scalar surface fluxes and identical scalar entrainment fluxes (at the top of the CBL) in both the LES and the mass-flux schemes.

Previous studies have shown that LES is able to provide detailed and realistic statistics for the CBL (Nieuwstadt et al. 1993). LES models are able to compute explicitly the most important lengths and timescales of the CBL (typically 25-1000 m and 10-10 000 $\mathrm{s}$, respectively). The small turbulence scales below the LES grid size of about $25 \mathrm{~m}$ have been found to have only a minor influence on the dynamics of the CBL, and therefore can be parameterized with a relatively simple LES subgrid scale model. Simulating a solid-lid CBL rather than an entraining CBL allows for integration toward a (quasi-)steady state of the scalar profiles, facilitating the comparison with (quasi-)steady state solutions of the mass-flux schemes (see also Brown 1996, who used a solid-lid CBL instead of an entraining CBL for similar reasons).

We categorize the differences between the mass-flux schemes in terms of the parameterization of the different component processes that appear in the plume-budget equations for scalars. Two components are relevant to transport of both nonreactive and reactive scalars, namely the parameterization of subplume fluxes and the parameterization of lateral-exchange processes between the updrafts and the downdrafts. Another component only pertains to reactive scalars, namely the parameterization of subplume covariances of reacting scalars. New parameterizations are proposed for the subplume fluxes and the subplume covariances.

In our view the attractiveness of mass-flux schemes for scalar transport in the CBL is threefold, compared to the first-order closure schemes that are currently used in large-scale atmospheric chemistry-transport models. First, nonlocal transport effects are clearly embodied in mass-flux schemes, which is attractive compared to the often used local first-order closure schemes (cf. Holtslag and Moeng 1991; Stull 1993). Second, the effects of a continuous distribution of fast chemical sources and sinks on the fluxes are taken into account (Fitzjarrald and Lenschow 1983; Schumann 1989; Sykes et al. 1994; Gao and Wesely 1994; Vilà-Guerau de Arellano et al. 1995; Galmarini et al. 1997; Verver et al. 1997). And third, the effects of horizontal segregation of reactive scalars on the mean reaction rates are represented (Schumann 1989; Vilà-Guerau de Arellano and Duynkerke 1993; Sykes et al. 1994; Beets et al. 1996; Verver et al. 1997; Molemaker and Vilà-Guerau de Arellano 1998).

We here briefly explain the two last mentioned advantages of mass-flux schemes for the transport of reactive scalars in the CBL. In the mass-flux schemes that we study in this paper we use the updraft-downdraft decomposition and treat both updraft and downdraft scalar quantities as prognostic variables. Therefore the mass-flux schemes considered here can be compared to higher-order (but not fully second-order) closure schemes in the sense that they contain two prognostic variables that provide a model for all higher-order moments. The mean scalar value and the scalar flux (a second moment) in a mass-flux scheme can for instance be written in terms of these updraft and downdraft quantities and can replace these as independent variables. The prognostic equations for the mean scalar values include a direct influence of the covariances, and the prognostic equations for the fluxes include a direct influence of the chemistry on the fluxes. In contrast to mass-flux schemes, first-order closure schemes do not implicitly include these two turbulence-chemistry interactions.

As already stated in the above, we do not evaluate complete mass-flux schemes in this paper, that is, we do not deal with questions concerning the parameterization of the mass flux and the updraft area fraction. Instead of this, we are concerned with evaluating the scalar transport-reaction characteristics of mass-flux schemes.

This paper is organized as follows. The LES of the solid-lid CBL, including the studied cases of scalar transport, is described in section 2. In section 3 we present the scalar plume-budget equations and the massflux schemes that can be derived from these. The LES results for the scalars appear in sections 4 and 5. In section 6 we present the results of the mass-flux schemes. Finally, we briefly summarize and discuss the results in section 7 .

\section{Description of LES and cases}

\section{a. LES of the solid-lid CBL}

The type of boundary layer studied here is a solidlid CBL (without entrainment of heat at the top of the CBL as opposed to the entraining CBL), which was also used for studying transport of reactive scalars by Beets et al. (1996) and Molemaker and Vilà-Guerau de Arellano (1998). The dynamics and thermodynamics of the solid-lid CBL was studied with LES by Schumann (1993). He found that LES compares favorably with laboratory experiments. Sorbjan (1996) studied the differences between solid-lid and entraining CBLs, of which the last one is more representative for the real atmosphere. Both convection experiments in tanks and numerical studies have shown that various turbulence statistics in the lower portion of the solid-lid CBL resemble those of the entraining CBL. Differences in the dynamics caused by the inclusion of entrainment of heat in the entraining CBL are reflected in a lower updraft area fraction $a$ in the upper-part of the CBL. Sorbjan (1996) found that $a$ is approximately 0.45 throughout the whole mixed layer for the solid-lid CBL but decreases to a minimum of 0.25 near the top of the mixed layer for the entraining CBL. Here it must be borne in mind that, as shown by Nieuwstadt et al. (1993), the minimum value of $a$ differs substantally among LES 
models of the entraining CBL: in their simulations the minimum value of $a$ varies roughly from 0.3 (model of Schumann) to 0.4 (model of Moeng). Furthermore, Sorbjan (1996) found that the average updraft vertical velocity $\bar{w}^{u}$ is almost the same for both types of CBL at every height.

Since the aim of this paper is the study of transport of dynamically passive scalars (with varying chemical reaction rates) in the CBL, we may exploit the ease with which fixed fluxes can be implemented at the top of the solid-lid CBL (identical to the top of the LES domain). The flux at the top is zero for potential temperature and zero or a finite constant for other scalars of interest. According to the results shown in Sorbjan (1996) the second (and third) moments in the surface layer (and for some variables also in the lower mixed layer) do not differ between the solid-lid and the entraining CBL. We have verified that conclusions reached in this study concerning mass-flux characteristics of second moments involving scalars in the solid-lid CBL can be extrapolated to the lower parts of the entraining CBL (surface layer and lower mixed layer) but certainly not to the entrainment layer at the top of the entraining CBL. The study of entrainment processes at the top of the CBL is not part of this work.

The algorithm for the large-eddy simulations performed in this study has been derived from a model used in earlier studies (Nieuwstadt and Brost 1986; van Haren and Nieuwstadt 1989; Nieuwstadt et al. 1993) and was previously used in Beets et al. (1996). We will describe the LES model here. The model is based on the Navier-Stokes equations for the velocities $u_{i}$, pressure $p$, potential temperature $\theta$, and other (nonreactive or reactive) scalars $s_{l}$ in the Boussinesq approximation. The LES model separates the flow and concentration field into a large-scale (resolved) indicated by ${ }^{\wedge}$ and $a$ small-scale (unresolved) field indicated by ", for example, $u_{i}=\widehat{u_{i}}+u_{i}^{\prime \prime}$. As is usual, it is assumed that this decomposition satisfies the Reynolds rules. Substitution of the decomposition in the Navier-Stokes equations then leads to the following set of prognostic equations for the resolved motions:

$$
\begin{aligned}
\frac{\partial \widehat{u_{i}}}{\partial x_{i}}= & 0, \\
\frac{\partial \widehat{u_{i}}}{\partial t}= & -\frac{\partial \widehat{u_{j}} \widehat{u_{i}}}{\partial x_{j}}-\frac{\partial \hat{\pi}}{\partial x_{i}}+\frac{g}{T_{r}}\left(\hat{\theta}-T_{r}\right) \delta_{i 3} \\
& +\frac{\partial\left(\widehat{u_{j}^{\prime \prime} u_{i}^{\prime \prime}}-\frac{2}{3} e \delta_{i j}\right)}{\partial x_{j}}, \\
\frac{\partial \hat{\theta}}{\partial t}= & -\frac{\partial \widehat{u_{j}} \hat{\theta}}{\partial x_{j}}-\frac{\partial \widehat{u_{j}^{\prime \prime} \theta^{\prime \prime}}}{\partial x_{j}}, \text { and }
\end{aligned}
$$

$$
\frac{\partial \widehat{s_{l}}}{\partial t}=-\frac{\partial \widehat{u_{j}} \widehat{s_{l}}}{\partial x_{j}}-\frac{\partial \widehat{u_{j}^{\prime \prime} s_{l}^{\prime \prime}}}{\partial x_{j}}+\widehat{S_{s_{l}}}+\widehat{S_{s_{l}}^{\prime \prime}}
$$

where the modified pressure $\hat{\pi}=\left(\hat{p}-p_{0}\right) / \rho_{r}+2 e / 3$ and $e=\frac{1}{2} \widehat{u_{i}^{\prime \prime} u_{i}^{\prime \prime}}$ is the subgrid scale kinetic energy. The quantity $S_{s_{l}}$ represents sources and sinks of the scalars $s_{l}$. In our LES model the constant reference density $\rho_{r}$ is taken equal to $1 \mathrm{~kg} \mathrm{~m}^{-3}$, the gravitational acceleration $g=9.8 \mathrm{~m} \mathrm{~s}^{-2}$, and the reference temperature $T_{r}=300$ K. The pressure $p_{0}$ can be found from

$$
p_{0}(z)=p_{s} \exp \left(-\int_{0}^{z} \frac{g}{R T_{r}} d z\right),
$$

with $p_{s}$ the surface pressure. Since the Reynolds number Re of the simulated flow is effectively infinite, the kinematic viscosity $\nu$, the thermal diffusivity $\kappa$, and Fickian diffusion coefficients $\mathcal{D}_{s_{k}}$ are effectively zero and hence do not appear in (2.2)-(2.4). No-slip and free-slip boundary conditions are prescribed at the surface and top of the domain, respectively. The surface roughness length $z_{0}$ is set to $0.16 \mathrm{~m}$. The boundary layer height $z_{i}$ $=1500 \mathrm{~m}$, the convective velocity scale $w_{*} \equiv\left(g \overline{w^{\prime} \theta^{\prime}}{ }_{0} z_{i} /\right.$ $\left.T_{r}\right)^{1 / 3}=1.5 \mathrm{~m} \mathrm{~s}^{-1}$, and $\overline{w^{\prime} \theta^{\prime}}{ }_{0}=0.069 \mathrm{~K} \mathrm{~m} \mathrm{~s}^{-1}$ the surface potential temperature flux. The resulting convective timescale is $t_{*}=z_{i} / w_{*}=1000 \mathrm{~s}$.

As will be discussed in the next subsection, in this paper we consider chemical sink terms for two species (labeled $l$ and $m$ ) reacting away in a binary reaction with reaction rate coefficient $k_{l m}^{\prime}$. The resolved and subgrid scale source and sink terms in the studied chemistry cases read

$$
\begin{aligned}
& \widehat{S_{l}}=\widehat{S_{m}}=-k_{l m}^{\prime} \widehat{s_{l}} \widehat{s_{m}}, \quad \text { and } \\
& \widehat{S_{l}^{\prime \prime}}=\widehat{S_{m}^{\prime \prime}} \equiv-k_{l m}^{\prime} \widehat{s_{l}^{\prime \prime} s_{m}^{\prime \prime}} .
\end{aligned}
$$

The equations for the resolved quantities (2.1)-(2.4) are solved explicitly. The subgrid scale Reynolds stresses, subgrid scale temperature flux, subgrid-scale scalar fluxes, and subgrid-scale covariances that appear in these equations are parametrized as a function of the resolved quantities, the normal stresses, and a timescale of subgrid scale turbulence $\tau$ according to

$\widehat{u_{i}^{\prime \prime} u_{j}^{\prime \prime}}=-C_{1} \tau\left(\widehat{u_{i}^{\prime \prime} u_{i}^{\prime \prime}} \frac{\partial \widehat{u_{j}}}{\partial x_{i}}+\widehat{u_{j}^{\prime \prime} u_{j}^{\prime \prime}} \frac{\partial \widehat{u_{i}}}{\partial x_{j}}\right) \quad i \neq j$,

$\widehat{u_{i}^{\prime \prime} \theta^{\prime \prime}}=-C_{2} \tau\left(\widehat{u_{i}^{\prime \prime} u_{i}^{\prime \prime}} \frac{\partial \hat{\theta}}{\partial x_{i}}\right)$

$\widehat{u_{i}^{\prime \prime} s_{l}^{\prime \prime}}=-C_{2} \tau\left(\widehat{u_{i}^{\prime \prime} u_{i}^{\prime \prime}} \frac{\partial \widehat{s_{l}}}{\partial x_{i}}\right), \quad$ and

$\widehat{s_{l}^{\prime \prime} s_{m}^{\prime \prime}}=-C_{3} \tau\left(\widehat{u_{i}^{\prime \prime} s_{l}^{\prime \prime}} \frac{\partial \widehat{s_{m}}}{\partial x_{i}}+\widehat{u_{i}^{\prime \prime} s_{m}^{\prime \prime}} \frac{\partial \widehat{s_{l}}}{\partial x_{i}}\right)$

where $\tau$ is the ratio of the characteristic grid size $\Delta$ and the square root of the subgrid scale kinetic energy $e$. A 
modeled conservation equation for all normal stresses is solved explicitly. The constants $C_{1}, C_{2}$, and $C_{3}$ in (2.8)-(2.11) are derived from inertial-subrange theory and can be expressed as

$$
\begin{aligned}
C_{1} & =\frac{l_{f}}{2 \pi \Delta}(1.5 \alpha)^{-1.5}, \\
C_{2} & =\frac{\alpha}{\beta} C_{1}, \text { and } \\
C_{3} & =\frac{\beta}{\alpha} C_{1},
\end{aligned}
$$

where $\alpha$ and $\beta$ are constants which appear in the expressions for the inertial subrange energy and concentration variance spectra. The following values that are typical for these constants are used: $\alpha=1.5$ and $\beta=$ 0.7. The filter length scale $l_{f}$ is taken equal to twice the characteristic grid size $\left(l_{f}=2 \Delta\right)$.

The governing equations for the resolved field are solved explicitly using a finite-volume technique. All terms in the filtered momentum equations are discretized using straightforward second-order central differences, except for the advective terms that are discretized using the method of Piacsek and Williams (1970). The leapfrog scheme, with an Asselin filter to prevent decoupling of odd and even time levels, is used for the time integration of the momentum equations. Conservation of mass is obtained by solving a diagnostic equation for the pressure. For the advection and diffusion of temperature and the other scalars we use the limited $\kappa=$ $\frac{1}{3}$ scheme (Koren 1993) for the spatial discretization and a two-stage Runge-Kutta method for the time integration (Hundsdorfer et al. 1995). For the time advancement of chemistry we use the routine Twostep (Verwer and Simpson 1995) and straightforward second-order central differences for the discretization of the subgrid scale covariance. The entire numerical discretization for temperature and the other scalars satisfies three important properties: it is conservative, positive, and monotone.

The grid used has $130 \times 130 \times 66$ grid points in the horizontal and vertical directions, respectively, representing a $6.0 \mathrm{~km} \times 6.0 \mathrm{~km} \times 1.5 \mathrm{~km}$ physical domain, thus employing a higher resolution than in previous studies with the LES model. The time step in the model is approximately $0.6 \mathrm{~s}$. A grid domain of $32 \times 32 \times$ 30 was used by Sorbjan (1996), but although the associated resolution is generally considered to be sufficient for the representation of basic turbulence characteristics of the CBL, we use a relatively high spatial resolution to minimize the LES unresolved covariance contribution to the chemical sink term and to be able to perform spectral investigations on very small scales. The unresolved covariance contribution is nearly zero in the bulk and much smaller than the resolved covariance near the bottom and top boundaries (this can also be checked by comparing Figs. 7a and 8).

\section{b. Cases}

For the nonreactive case, called BUTD, we have introduced in our LES a passive bottom-up diffusing scalar (BU) and a passive top-down diffusing scalar (TD). The flux of BU at the top of the CBL is put to zero, as is the flux of TD at the bottom. Choosing a flux scale $F_{*}$, the constant input fluxes of BU and TD are set to $F_{*}$ and $-F_{*}$, respectively. Using the convective velocity scale $w_{*}$, a scalar-value scale can then be defined as $s_{*}$ $=F_{*} / w_{*}$. If later in this paper no units are assigned to specific quantities, it is assumed that these quantities have been made dimensionless using the scales defined here.

The reactive cases, called $\mathrm{AB} 1, \mathrm{AB} 2, \mathrm{AB} 3$, and $\mathrm{AB} \infty$, are of the following type: they consist of bottom-up (A) and top-down (B) scalars reacting away in a secondorder reaction $(\mathrm{A}+\mathrm{B} \rightarrow \mathrm{C})$.

To illustrate the effect of horizontal segregation on the mean reaction rate we write the sink term for species $\mathrm{A}$ and $\mathrm{B}$ due to the reaction $\mathrm{A}+\mathrm{B} \rightarrow \mathrm{C}$ as

$$
\begin{aligned}
S & =-k_{\mathrm{A}+\mathrm{B} \rightarrow \mathrm{C}}^{\prime} \overline{s_{\mathrm{A}} s_{\mathrm{B}}} \\
& =-k_{\mathrm{A}+\mathrm{B} \rightarrow \mathrm{C}}^{\prime}\left(\overline{s_{\mathrm{A}}} \overline{s_{\mathrm{B}}}+\overline{s_{\mathrm{A}}^{\prime} s_{\mathrm{B}}^{\prime}}\right),
\end{aligned}
$$

where $s_{\mathrm{A}}$ and $s_{\mathrm{B}}$ are the scalar values (concentrations) of chemical species A and B. Here and throughout the rest of this paper overbars denote horizontal ensemble averages and primes denote fluctuations from these averages. The intensity of segregation, defined as

$$
I_{s}=\frac{\overline{s_{\mathrm{A}}^{\prime} s_{\mathrm{B}}^{\prime}}}{\overline{s_{\mathrm{A}}} \overline{s_{\mathrm{B}}}},
$$

is a measure of the importance of the covariance term $\overline{s_{\mathrm{A}}^{\prime} s_{\mathrm{B}}^{\prime}}$ appearing in (2.15). The intensity of segregation obeys the inequality $I_{\mathrm{s}} \geq-1$. The reaction-rate coefficient $k^{\prime}$ that appears in (2.15) can be made nondimensional in the following way:

$$
k_{\mathrm{A}+\mathrm{B} \rightarrow \mathrm{C}}=s_{*} t_{*} k_{\mathrm{A}+\mathrm{B} \rightarrow \mathrm{C}}^{\prime}
$$

The nondimensional reaction-rate coefficient is also called the "flux Damköhler number." We expect the largest segregation effects for fast chemical reactions ( $k$ $\gg 1$ ), and we will vary the flux Damköhler number from zero (nonreactive case) to infinity. The three nondimensional reaction-rate coefficients used in cases $\mathrm{AB} 1, \mathrm{AB} 2$, and $\mathrm{AB} 3$ are 0.2, 1.0, and 5.0, respectively.

As in the nonreactive case, for all reactive cases only two boundary fluxes are nonzero. These are the constant fluxes of scalars A and B into the boundary layer with values $F_{*}$ at the bottom and $-F_{*}$ at the top, respectively. The other two flux boundary conditions are put to zero. The concentrations of scalars A and B reach a steady state due to the presence of the chemical sink.

A rather special case is $\mathrm{AB} \infty$, for which one does not have to do a chemistry run nor a series of runs to approximate the infinite reaction rate limit. Instead, we can diagnose the $\mathrm{A}$ and $\mathrm{B}$ scalar fields for case $\mathrm{AB} \infty$ 
from the BU and TD scalar fields in the nonreactive case. As was also done by Schumann (1989), we may use $\left|s_{\mathrm{BU}}-s_{\mathrm{TD}}\right|$ as a substitute for $s_{\mathrm{A}}$ if $s_{\mathrm{BU}} \geq s_{\mathrm{TD}}\left(s_{\mathrm{B}}\right.$ is then set to zero) and for $s_{\mathrm{B}}$ if $s_{\mathrm{BU}}<s_{\mathrm{TD}}\left(s_{\mathrm{A}}\right.$ is then set to zero). In other words, we can regard scalars $A$ and $\mathrm{B}$ as diffusing from their respective sources at the bottom and top of the CBL (without reacting) to a highly complex reaction interface of zero thickness. At one side of this interface surface only scalar B is present and at the other side of this interface only scalar A. To defend the above outlined procedure for case $\mathrm{AB} \infty$, we show in appendix B that the scalars A and B become uncorrelated at the smallest scale that is resolved in the LES model when we increase the reaction-rate coefficient from moderate to infinite values. Correspondingly, the unresolved covariance contribution will become negligible for very high reaction-rate coefficients (as it is for nonreactive scalars).

Each LES run is started with a well-developed turbulent layer and varying initial scalar profiles (always with equal total amounts of both species present in the CBL). For cases with a chemical timescale smaller than the convective timescale the scalars come close to a steady state after several convective turnovers (turbulent mixing being the limiting factor). For slower chemistry, the dimensionless chemical timescale $k^{-1}$ determines the number of required turnovers. The averaging process is not started before it has been verified that the scalars have nearly reached their steady-state values (in case BUTD the scalars will reach a quasi-steady state characterized by a linear flux profile). Then the averaging takes place using eight consecutive snapshots (with a time separation of $0.25 t_{*}$ ) during an integration of length $2 t_{*}$. The only exception to this procedure is case $\mathrm{AB} \infty$ : we perform the calculations for this case only on the final field of case BUTD. Therefore the profiles for case $\mathrm{AB} \propto$ are somewhat less smooth than the other profiles.

\section{Scalar plume budgets}

\section{a. Basic equations}

Firstly, we introduce the convective mass flux $M$ :

$$
M=\rho a\left(\bar{w}^{u}-\bar{w}\right),
$$

with $a$ the updraft area fraction, $\bar{w}^{u}$ the updraft velocity, and $\bar{w}$ the mean vertical velocity. As stated before, we take the density $\rho=1 \mathrm{~kg} \mathrm{~m}^{-3}$ and we therefore do not explicitly include $\rho$ in our equations below.

We use the following decomposition of the scalar flux:

$$
\begin{aligned}
\overline{w^{\prime} s^{\prime}}= & a\left(\bar{w}^{u}-\bar{w}\right)\left(\bar{s}^{u}-\bar{s}\right)+(1-a)\left(\bar{w}^{d}-\bar{w}\right)\left(\bar{s}^{d}-\bar{s}\right) \\
& +a \overline{w^{\prime} s^{\prime}}{ }^{u}+(1-a) \overline{w^{\prime} s^{\prime}} d \\
= & M\left(\bar{s}^{u}-\bar{s}^{d}\right)+a \overline{w^{\prime} s^{\prime}}{ }^{u}+(1-a) \overline{w^{\prime} s^{\prime}}{ }^{d}
\end{aligned}
$$

as is done by Siebesma and Cuijpers (1995). The indices $u$ and $d$ indicate that the averaging areas consist of updrafts and downdrafts, respectively. In decomposition (3.2) we can distinguish between mass-flux (also called "top hat") and subplume contributions to the total flux, and the decomposition is exact. The terms ${\overline{w^{\prime} s^{\prime}}}^{u}$ and $\overline{w^{\prime} s^{\prime}} d$ represent fluxes associated with subplume correlations of vertical velocity and scalar quantities and are defined as ${\overline{w^{\prime} s^{\prime}}}^{u} \equiv \overline{\left(w-\bar{w}^{u}\right)\left(s-\bar{s}^{u}\right)}{ }^{u}$ and ${\overline{w^{\prime} s^{\prime}}}^{d} \equiv$ ${\left.\overline{\left(w-\bar{w}^{d}\right.}\right)\left(s-\bar{s}^{d}\right)}^{d}$.

Using $a$ and $M$ we can write the following massconservation equation, or continuity equation:

$$
\frac{\partial a}{\partial t}=-\frac{\partial M}{\partial z}+E_{m}-D_{m},
$$

where, following Siebesma (1997), we have used the gross lateral mass-exchange rates $E_{m}$ and $D_{m}$, also called entrainment and detrainment rates, respectively (the terms entrainment and detrainment are defined relative to the updraft). These are defined as

$$
\begin{aligned}
& E_{m} \equiv-\frac{1}{A} \int_{\mathbf{n} \cdot\left(\mathbf{u}-\mathbf{u}_{i}\right)<0} \mathbf{n} \cdot\left(\mathbf{u}-\mathbf{u}_{i}\right) d l, \quad \text { and } \\
& D_{m} \equiv \frac{1}{A} \int_{\mathbf{n} \cdot\left(\mathbf{u}-\mathbf{u}_{i}\right)>0} \mathbf{n} \cdot\left(\mathbf{u}-\mathbf{u}_{i}\right) d l,
\end{aligned}
$$

where the integrals are over specific segments of all the interfaces between updrafts and downdrafts in a horizontal domain, $A, \mathbf{n}$ is an outward directed unit normal vector at the interface (outward is defined relative to the updraft), $\mathbf{u}$ is the $3 \mathrm{D}$ flow velocity field, and $\mathbf{u}_{i}$ is the 3D interface velocity field. This formulation is generally valid for any interface. Since in the case of an interface between updrafts and downdrafts $w=w_{i}=0$ the integrals contain only horizontal contributions.

The exact updraft and downdraft scalar plume-budget equations can be written, again following Siebesma (1997), as

$$
\begin{aligned}
\frac{\partial a \bar{s}^{u}}{\partial t}= & -\frac{\partial M \bar{s}^{u}}{\partial z}+\frac{1}{A} \oint_{\text {interface }} \mathbf{n} \cdot\left(\mathbf{u}-\mathbf{u}_{i}\right) s d l \\
& -\frac{\partial a \overline{w^{\prime} s^{\prime}} u}{\partial z}+a\left(S_{u}+S_{u, \text { subplume }}\right),
\end{aligned}
$$

and

$$
\begin{aligned}
\frac{\partial(1-a) \bar{s}^{d}}{\partial t}= & +\frac{\partial M \bar{s}^{d}}{\partial z}-\frac{1}{A} \oint_{\text {interface }} \mathbf{n} \cdot\left(\mathbf{u}-\mathbf{u}_{i}\right) s d l \\
& -\frac{\partial(1-a){\overline{w^{\prime} s^{\prime}}}^{d}}{\partial z}+(1-a)\left(S_{d}+S_{d \text {,subplume }}\right) .
\end{aligned}
$$

The term containing the contour integral represents lateral-exchange processes and the last terms in (3.6) and (3.7) correspond to sources and sinks, being subdivided 
into a plume mean and a subplume part (to be discussed below).

Below we will first discuss various parameterizations for the two types of subplume contributions to the plume-budget equations. This is followed by a discussion of parameterizations for the contour integral representing lateral-exchange processes. At the end of this section we will define the five mass-flux schemes for scalar transport that are studied in this paper as different combinations of the various parameterizations presented below.

\section{b. Subplume contributions to the plume budget equations}

\section{1) SubPlume FluXes $\overline{w^{\prime} s^{\prime}}$ AND $\overline{w^{\prime} s^{\prime}} d$}

In the past it has been found by Businger and Oncley (1990) for the surface layer and by Young (1988a), Schumann and Moeng (1991a), Wyngaard and Moeng (1992), and de Laat and Duynkerke (1998) for the CBL that the top-hat contribution to the flux, $M\left(\bar{s}^{u}-\bar{s}^{d}\right)$, is a constant fraction $\kappa_{w s}$ of the total flux, in formula form:

$$
\overline{w^{\prime} s^{\prime}} \approx \kappa_{w s}^{-1} M\left(\bar{s}^{u}-\bar{s}^{d}\right) \text {. }
$$

We can give a theoretical estimate of $\kappa_{w s}$, as shown by Wyngaard and Moeng (1992), provided that the joint probability density function (pdf) $P\left(w^{\prime}, s^{\prime}\right)$ of vertical velocity and scalar fluctuations is a Gaussian function. In that case, $\kappa_{w s}=4 /(2 \pi)=0.64$.

In the mass-flux schemes for scalar transport studied in this paper the subplume fluxes are treated in three different ways. First, they can be assumed to be zero:

$$
{\overline{w^{\prime} s^{\prime}}}^{u}={\overline{w^{\prime} s^{\prime}}}^{d}=0 \text {. }
$$

For a given total scalar flux and a given mass flux this will result in an overestimation of $\left(\bar{s}^{u}-\bar{s}^{d}\right)$ in (3.2) by a factor $\kappa_{w s}^{-1}$, according to (3.8).

Second, the subplume fluxes can be assumed to be proportional to the gross plume-scale top-hat contributions $M s^{u}$ and $M \bar{s}^{d}$, respectively, as implicitly done by Randall et al. (1992):

$$
\begin{aligned}
& {\overline{w^{\prime} s^{\prime}}}^{u}=\frac{1-\kappa_{w s}}{a \kappa_{w s}} M \bar{s}^{u}, \quad \text { and } \\
& {\overline{w^{\prime} s^{\prime}}}^{d}=\frac{1-\kappa_{w s}}{(1-a) \kappa_{w s}} M \bar{s}^{d} .
\end{aligned}
$$

In this parameterization it is assumed that the subplume fluxes can be absorbed into the top-hat flux terms by using $\kappa_{w s}^{-1} M$ instead of $M$ in (3.2). Equations (3.10) and (3.11) are consistent with (3.8).

Third, we propose a new subplume fluxe parameterization based on the results shown in Figs. 4a,b (to be discussed in section 4). This new parameterization makes use of the fact that purely bottom-up diffusing scalars only have a subplume flux in the updraft and purely top-down diffusing scalars only have a subplume flux in the downdraft. According to the superposition hypothesis of Wyngaard and Brost (1984), any nonreactive passive scalar can be written as a linear combination of bottom-up and top-down diffusing scalar fields:

$$
s=\alpha s_{\mathrm{BU}}+\beta s_{\mathrm{TD}},
$$

where the scalars BU and TD are assumed to have equal but opposite input fluxes for BU at the bottom of the CBL and for TD at the top of the CBL. If we assume quasi-steady conditions, the fractional contributions of both component fields to the total flux at a certain height, $\gamma_{\mathrm{BU}}$ and $\gamma_{\mathrm{TD}}$, are

$$
\gamma_{\mathrm{BU}} \equiv 1-\gamma_{\mathrm{TD}}=\frac{|\alpha|\left(1-\frac{z}{z_{i}}\right)}{|\alpha|\left(1-\frac{z}{z_{i}}\right)+|\beta| \frac{z}{z_{i}}} .
$$

The new subplume-fluxes parameterization reads

$$
\begin{aligned}
& {\overline{w^{\prime} s^{\prime}}}^{u}=\gamma_{\mathrm{BU}} \frac{1-\kappa_{w s}}{a \kappa_{w s}} M\left(\bar{s}^{u}-\bar{s}^{d}\right), \quad \text { and } \\
& {\overline{w^{\prime} s^{\prime}}}^{d}=\gamma_{\mathrm{TD}} \frac{1-\kappa_{w s}}{(1-a) \kappa_{w s}} M\left(\bar{s}^{u}-\bar{s}^{d}\right),
\end{aligned}
$$

which is also consistent with (3.8). For scalars that have a continuous distribution of sources and sinks in the $\mathrm{CBL}$, the flux profile can deviate from linearity and the decomposition of the scalar field in bottom-up and topdown components is not strictly valid anymore. However, we will apply (3.14) and (3.15) also in our reactive cases, since we also found for these cases that bottomup species have subplume fluxes in the updraft only and top-down species have subplume fluxes in the downdraft only.

\section{2) SubPlume COVARIANCES ${\overline{s_{\mathrm{A}}^{\prime} s_{\mathrm{B}}^{\prime}}}^{u}$ AND ${\overline{s_{\mathrm{A}}^{\prime} s_{\mathrm{B}}^{\prime}}}^{d}$}

In the same manner as we did in (3.2) for the flux, we write the following decomposition of the covariance:

$$
\begin{aligned}
& \overline{s_{\mathrm{A}}^{\prime} s_{\mathrm{B}}^{\prime}} \\
& =a\left(\overline{s_{\mathrm{A}}}{ }^{u}-\overline{s_{\mathrm{A}}}\right)\left({\overline{s_{\mathrm{B}}}}^{u}-\overline{s_{\mathrm{B}}}\right)+(1-a)\left({\overline{s_{\mathrm{A}}}}^{d}-\overline{s_{\mathrm{A}}}\right)\left({\overline{s_{\mathrm{B}}}}^{d}-\overline{s_{\mathrm{B}}}\right) \\
& +a{\overline{s_{\mathrm{A}}^{\prime} s_{\mathrm{B}}^{\prime}}}^{u}+(1-a) \overline{s_{\mathrm{A}}^{\prime} s_{\mathrm{B}}^{\prime}} d \text {. }
\end{aligned}
$$

The first two terms on the rhs together constitute the top-hat contribution to the covariance and the last two terms contain the subplume covariances. These subplume covariances appear in the formulas for the subplume contributions to chemical sinks and sources, $S_{u \text { subplume }}$ and $S_{d, \text { subplume }}$. The subplume contributions are relevant only to sinks and sources related to secondorder chemical reactions. In the two plume-budget equations (3.6) and (3.7) the chemical sink terms for our type of reactive cases look as follows: 


$$
\begin{aligned}
& S_{u}=-k{\overline{s_{\mathrm{A}}}}^{u}{\overline{S_{B}}}^{u}, \\
& S_{d}=-k{\overline{s_{\mathrm{A}}}}^{d}{\overline{s_{B}}}^{d} \text {, }
\end{aligned}
$$

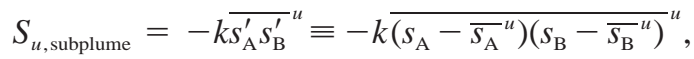

and

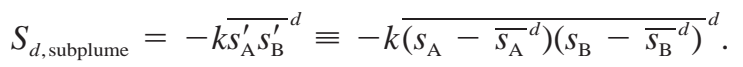

As we will show in this paper the subplume contributions to the total chemical sinks and sources are substantial, so they must be parameterized.

We will treat subplume covariances in two ways in the mass-flux schemes studied in this paper. First, we can assume them to be zero:

$$
{\overline{s_{\mathrm{A}}^{\prime} s_{\mathrm{B}}^{\prime}}}^{u}={\overline{s_{\mathrm{A}}^{\prime} s_{\mathrm{B}}^{\prime}}}^{d}=0 .
$$

For our reactive cases, the use of (3.21) will lead to underestimating the absolute value of the intensity of segregation $I_{\mathrm{s}}$ (it will be less negative) and consequently to overestimating the mean reaction rate, resulting in lower steady-state concentrations of scalars A and B.

Second, we propose a new parameterization for the subplume covariances based on the results shown in Fig. $7 \mathrm{~b}$ (to be discussed in section 5). Analogous to the fluxes, we assume that the top-hat contribution to the total covariance is a constant fraction $\kappa_{\mathrm{AB}}$ of the total covariance:

$$
\begin{aligned}
\overline{s_{\mathrm{A}}^{\prime} s_{\mathrm{B}}^{\prime}} & \approx \kappa_{\mathrm{AB}}^{-1}\left[a{\overline{s_{\mathrm{A}}}}^{u}{\overline{s_{\mathrm{B}}}}^{u}+(1-a) \overline{s_{\mathrm{A}}} d \overline{s_{\mathrm{B}}} d-\overline{s_{\mathrm{A}}} \overline{s_{\mathrm{B}}}\right] \\
& \equiv \kappa_{\mathrm{AB}}^{-1} \overline{s_{\mathrm{A}}^{\prime} s_{\mathrm{B}}^{\prime}}
\end{aligned}
$$

The value of $\kappa_{\mathrm{AB}}$ is approximately 0.25 : only $25 \%$ of the total covariance is resolved by the top-hat term. The experimental backing for this value of $\kappa_{\mathrm{AB}}$ is discussed extensively in section 5 (where Fig. $7 \mathrm{~b}$ is discussed) and appendix A. We propose the following parameterization for the subplume covariances on the basis of this result:

$$
\begin{aligned}
& {\overline{s_{\mathrm{A}}^{\prime} s_{\mathrm{B}}^{\prime}}}^{u}=\frac{1-\kappa_{\mathrm{AB}}}{2 a \kappa_{\mathrm{AB}}} \overline{s_{\mathrm{A}}^{\prime} s_{\mathrm{B}}^{\prime}}{ }_{\text {top-hat }}, \quad \text { and } \\
& {\overline{s_{\mathrm{A}}^{\prime} s_{\mathrm{B}}^{\prime}}}^{d}=\frac{1-\kappa_{\mathrm{AB}}}{2(1-a) \kappa_{\mathrm{AB}}} \overline{s_{\mathrm{A}}^{\prime} s_{\mathrm{B}}^{\prime}}
\end{aligned}
$$

limiting the values of ${\overline{s_{\mathrm{A}}^{\prime} s_{\mathrm{B}}^{\prime}}}^{u}$ and ${\overline{s_{\mathrm{A}}^{\prime} s_{\mathrm{B}}^{\prime}}}^{d}$ on the negative side to $-{\overline{s_{\mathrm{A}}}}^{u}{\overline{S_{\mathrm{B}}}}^{u}$ and $-{\overline{s_{\mathrm{A}}}}^{d}{\overline{s_{\mathrm{B}}}}^{d}$, respectively. Equations (3.23) and (3.24) are similar to (3.14) and (3.15) for the subplume fluxes, except for the fact that in (3.23) and (3.24) it is assumed that the contributions of the updraft and the downdraft subplume covariances to the total covariance are equal. This is why the factor 2 appears in the denominators of (3.23) and (3.24). As said in the above the parameterization proposed here for the subplume covariances will be defended on the basis of LES results in section 5 .

\section{c. Lateral-exchange terms}

The contour integral for the lateral scalar exchange processes, appearing in (3.6) and (3.7), can be written as a sum of two terms:

$$
\frac{1}{A} \oint_{\text {interface }} \mathbf{n} \cdot\left(\mathbf{u}-\mathbf{u}_{i}\right) s d l=\left(E_{m}-D_{m}\right) \bar{s}^{i}+a_{i} \overline{v_{R}^{\prime} s^{\prime}}{ }^{i},
$$

where ${ }_{S}^{i}$ is the mean scalar value at the interface between updrafts and downdrafts, $a_{i}$ is the perimeter/area ratio of the updrafts, $v_{R}$ is shorthand for $\mathbf{n} \cdot\left(\mathbf{u}-\mathbf{u}_{i}\right)$, and the primes denote fluctuations relative to the interface average, which is denoted by the overbar with index $i$. The first term on the rhs of (3.25) is an interface-scale term and the second term is a subinterface scale term.

In our mass-flux schemes for scalar transport we will use two parameterizations of the lateral-exchange terms. Both parameterizations provide a closure for the rhs of (3.25) in terms of the updraft and downdraft quantities $\bar{s}^{u}$ and $\bar{s}^{d}$.

First, we follow Chatfield and Brost (1987) and write

$$
\left(E_{m}-D_{m}\right) \bar{s}^{i}+a_{i}{\overline{v_{R}^{\prime} s^{\prime}}}^{i}=r_{d} \bar{s}^{d}-r_{u} \bar{s}^{u},
$$

with

$$
\begin{aligned}
& r_{u}=\max \left\{-\frac{\partial M}{\partial z}, 0\right\}, \text { and } \\
& r_{d}=\max \left\{\frac{\partial M}{\partial z}, 0\right\} .
\end{aligned}
$$

In this parameterization only net advection occurs from one draft to the other (with the direction depending on the sign of $\partial M / \partial z$ ). This can be regarded as a parameterization of the interface-scale lateral exchange only, substituting $\bar{s}^{u}$ and $\bar{s}^{d}$ for $\bar{s}^{i}$ depending on the direction of the net mass exchange.

Second, we include the parameterization used extensively in cumulus schemes (e.g., Arakawa and Schubert 1974; Tiedtke 1989):

$$
\left(E_{m}-D_{m}\right) \bar{s}^{i}+a_{i}{\overline{v_{R}^{\prime} s^{\prime}}}^{i}=E_{s} \bar{s}^{d}-D_{s} \bar{s}^{u},
$$

with $E_{s}$ and $D_{s}$ being "scalar entrainment" and "scalar detrainment" quantities. The difference between (3.26) and (3.29) is that $E_{s}$ and $D_{s}$-provided that they are assigned positive values-are related to gross exchange, $E_{s}$ to advection from downdraft to updraft, and $D_{s}$ to simultaneous advection from updraft to downdraft. Thus, contrary to (3.26), (3.29) does include a parameterization for the subinterface-scale lateral-exchange process. The plume-budget equations (3.6) and (3.7) now become 
TABLE 1. Composition of mass-flux schemes for scalar transport with reference to equations in this paper and with reference to corresponding literature: Chatfield and Brost 1987 (CB87), Randall et al. 1992 (RSM92), and Siebesma and Cuijpers 1995 (SC95).

\begin{tabular}{|c|c|c|c|}
\hline Scheme & $\begin{array}{l}\text { Lateral-exchange terms } \\
\left(E_{m}-D_{m}\right) \bar{s}^{i}+a_{i}{\overline{v_{R}^{\prime} s^{\prime}}}^{i}\end{array}$ & $\begin{array}{l}\text { Subplume fluxes } \\
\bar{w}^{\prime} s^{\prime} \\
\end{array}$ & $\begin{array}{l}\text { Subplume covariances } \\
\frac{s_{\mathrm{A}}^{\prime} s_{\mathrm{B}}^{\prime}}{u} \text { and } \overline{s_{\mathrm{A}}^{\prime} s_{\mathrm{B}}^{\prime}} d\end{array}$ \\
\hline MF1 & $\begin{array}{c}(3.29),(3.33), \text { and }(3.34) \\
\text { SC95 }\end{array}$ & $\begin{array}{l}(3.14) \text { and }(3.15) \\
\text { this paper (new) }\end{array}$ & $\begin{array}{l}\text { (3.23) and (3.24) } \\
\text { this paper (new) }\end{array}$ \\
\hline MF2 & $\begin{array}{c}(3.26),(3.27), \text { and }(3.28) \\
\text { CB87 }\end{array}$ & $\begin{array}{l}(3.14) \text { and }(3.15) \\
\text { this paper (new) }\end{array}$ & $\begin{array}{l}\text { (3.23) and (3.24) } \\
\text { this paper (new) }\end{array}$ \\
\hline MF3 & $\begin{array}{c}(3.26),(3.27), \text { and }(3.28) \\
\text { CB87 }\end{array}$ & $\begin{array}{l}\text { (3.10) and (3.11) } \\
\text { RSM92 }\end{array}$ & $\begin{array}{l}\text { (3.23) and (3.24) } \\
\text { this paper (new) }\end{array}$ \\
\hline MF4 & $\begin{array}{c}(3.26),(3.27), \text { and }(3.28) \\
\text { CB87 }\end{array}$ & $\begin{array}{l}\text { (3.9) } \\
\text { no ref. (zero) }\end{array}$ & $\begin{array}{l}(3.21) \\
\text { no ref. (zero) }\end{array}$ \\
\hline MF5 & $\begin{array}{c}(3.26),(3.27), \text { and }(3.28) \\
\text { CB87 }\end{array}$ & $\begin{array}{c}\text { (3.10) and (3.11) } \\
\text { RSM92 }\end{array}$ & $\begin{array}{l}(3.21) \\
\text { no ref. (zero) }\end{array}$ \\
\hline
\end{tabular}

$$
\begin{aligned}
\frac{\partial a \bar{s}^{u}}{\partial t}= & -\frac{\partial M \bar{s}^{u}}{\partial z}+E_{s} \bar{s}^{d}-D_{s} \bar{s}^{u}-\frac{\partial a \overline{w^{\prime} s^{\prime}}}{\partial z} \\
& +a\left(S_{u}+S_{u, \text { subplume }}\right), \text { and } \\
\frac{\partial(1-a) \bar{s}^{d}}{\partial t}= & +\frac{\partial M \bar{s}^{d}}{\partial z}-E_{s} \bar{s}^{d}+D_{s} \bar{s}^{u}-\frac{\partial(1-a){\overline{w^{\prime} s^{\prime}}}^{d}}{\partial z} \\
& +(1-a)\left(S_{d}+S_{d, \text { subplume }}\right) .
\end{aligned}
$$

As Young (1988b) and Schumann and Moeng (1991b) did, we can determine $\left(E_{s} \bar{s}^{d}-D_{s} \bar{s}^{u}\right)$ as a residual term of either (3.30) or (3.31). However, it is also possible to determine unique profiles of $E_{s}$ and $D_{s}$, provided that we impose some constraint. It is convenient to impose, as is implicitly done by Siebesma and Cuijpers (1995),

$$
E_{s}-D_{s}=E_{m}-D_{m} .
$$

Substituting the continuity equation (3.3) in (3.30) and (3.31), and noting that we have $\bar{w}=0$ in our LES, results in

$$
\begin{aligned}
E_{s}\left(\bar{s}^{u}-\bar{s}^{d}\right)= & \bar{s}^{u} \frac{\partial M}{\partial z}-\frac{a \overline{w s}}{\partial z}-a \frac{\partial \bar{s}^{u}}{\partial t} \\
& +a\left(S_{u}+S_{u, \text { subplume }}\right), \text { and } \\
D_{s}\left(\bar{s}^{u}-\bar{s}^{d}\right)= & \bar{s}^{d} \frac{\partial M}{\partial z}+\frac{(1-a) \overline{w s}^{d}}{\partial z}+(1-a) \frac{\partial \bar{s}^{d}}{\partial t} \\
& -(1-a)\left(S_{d}+S_{d, \text { subplume }}\right) .
\end{aligned}
$$

One must be aware that the quantities $E_{s}$ and $D_{s}$ defined by (3.29) and (3.32) are not guaranteed to be scalarindependent. Also, $E_{s}$ and $D_{s}$ do not have to be positive. The closure assumption (3.29), in combination with determining $E_{s}$ and $D_{s}$ from (3.33) and (3.34), might even lead to plume-budget equations that do not have stable solutions (due to negative $E_{s}$ and $D_{s}$ ). In sections 4 and 5 we will determine the behavior of $E_{s}$ and $D_{s}$ for different types of scalars from LES. Also we will specify there which profiles of $E_{s}$ and $D_{s}$ are used in our most comprehensive mass-flux scheme for scalar transport.

\section{d. Mass-flux schemes for scalar transport}

Now we have come to the point where we can define the mass-flux schemes that we will evaluate in this study. The mass-flux schemes can be considered as composed of the basic scalar plume-budget equations (3.6) and (3.7) with different permutations of the parameterizations presented in the above for the subplume fluxes, the subplume covariances, and the lateral-exchange terms. As said before, the mass-flux schemes are run in an off-line mode.

In Table 1 we define five mass-flux schemes (MF1 up to MF5) for transport of nonreactive and reactive scalars in the CBL. The column pertaining to the subplume-covariance parameterization is only relevant to the reactive cases, so for the nonreactive case BUTD we effectively have four different mass-flux schemes (for this case MF5 is identical to MF3). We use the same vertical grid resolution in the mass-flux schemes as in the LES, namely 66 layers.

Since the new proposals for the parameterization of subplume fluxes and subplume covariances are based on LES results (to be presented in sections 4 and 5) and since the parameterization of lateral exchange according to Siebesma and Cuijpers (1995) also uses LES results as input, scheme MF1 has the closest resemblance to the exact plume-budget equations. Going down the list of schemes, less sophisticated parameterizations are used for the three component processes and we expect in general that the performance will become worse (although, as it turns out there can be "compensating errors" at play, resulting in a better performance of a relatively simple scheme compared to a relatively complex scheme).

\section{LES results for nonreactive scalars}

a. Scalar profiles $\overline{\mathrm{s}_{B U}}$ and $\overline{\mathrm{s}_{T D}}$

Updraft and downdraft profiles of bottom-up and topdown diffusing scalars BU and TD, obtained from the LES by way of conditional sampling (averaging over areas with respectively positive and negative vertical 

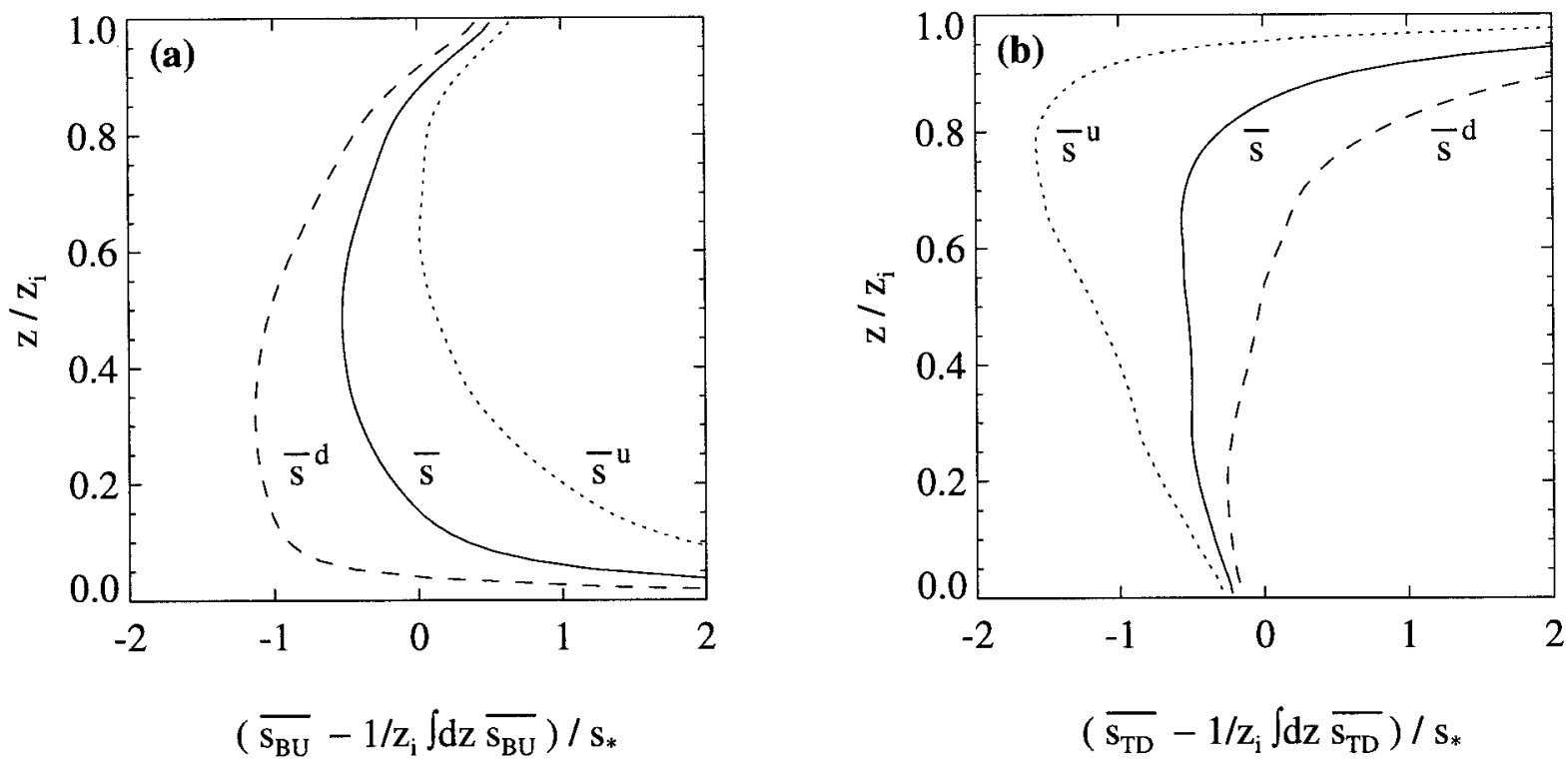

FIG. 1. Profiles of (a) bottom-up diffusing scalar BU and (b) top-down diffusing scalar TD, determined from LES; the steady deviation from the bulk value is plotted, calculated by subtracting the vertically integrated (and steadily increasing) scalar quantities from the vertical profiles. Mean, updraft, and downdraft values are shown.

velocities), are shown in Figs. 1a,b. The BU and TD scalar profiles are in quasi-steady state (with the gradients not changing in time), since there is a constant influx at either the bottom or the top boundary and there is no sink for each of the two scalars. Therefore we have subtracted the (steadily increasing) boundary layer averaged scalar values from the profiles before plotting them in Figs. 1a,b, respectively.

A striking (but not perfect) symmetry between bottom-up and top-down transport mechanisms exists in the solid-lid CBL. For both scalars we find countergradient fluxes near their respective zero-flux boundaries: a stronger countergradient flux near the top for the BU scalar and a weaker countergradient flux near the bottom for the TD scalar. The countergradient fluxes of the scalars are caused by the presence of relatively fast cores within both updrafts and downdrafts that quickly vertically transport the scalars through the CBL. In the entraining CBL we do not find a countergradient flux of top-down diffusing scalars, since there is less vertical symmetry in the entraining CBL.

\section{b. Fluxes $\overline{w^{\prime} s_{B U}^{\prime}}$ and $\overline{w^{\prime} s_{T D}^{\prime}}$}

The flux profiles for case BUTD plotted in Figs. 2a,b are nearly linear, which means that the scalar profiles in Figs. 1a,b are close to quasi-steady state.

In Figs. 3a,b the ratios of the top-hat contribution to the total flux $\kappa_{w s}$ are shown for bottom-up and top-down diffusing scalars, respectively. We find that the theoretical estimate (based on Gaussian assumptions) of $\kappa_{w s}$ $=0.64$ holds quite well for the BU and TD scalars, although the BU scalar shows a somewhat lower value of $\kappa_{w s}$ near the top, where the fluxes become small. A value of 0.6 for $\kappa_{w s}$ was also found before by Businger and Oncley (1990) from measurements in the surface layer for all stabilities, by Schumann and Moeng (1991a) and Wyngaard and Moeng (1992) from LES of the clear and stratocumulus-topped CBL and by de Laat and Duynkerke (1998) from measurements in the stratocumulus-topped CBL. For the solid-lid CBL we do not find the higher value of $\kappa_{w s}$ for the TD scalar that was found before by Wyngaard and Moeng (1992) for the entraining CBL (which is again due to the fact that the entraining CBL is less symmetrical in the vertical).

In Figs. 4a,b the LES results are shown for the decomposition of the total fluxes as given in (3.2). It turns out that the subplume flux of scalar BU is located only in the updraft and that the subplume-flux of scalar TD is located only in the downdraft. This fact was used in our subplume flux parameterization (3.14) and (3.15). Although Chatfield and Brost (1987) and Hunt et al. (1988) refer only to updrafts and not to downdrafts when dealing with subplume fluxes, our result that the subplume flux of scalar TD is carried almost completely by the downdrafts shows the importance of subdowndraft fluxes for scalar fields which have a significant top-down component. This is also evident in the results presented by Young (1988b) for the vertical velocity budget in the downdrafts present in the entraining CBL.

\section{c. Lateral entrainment and detrainment rates $E_{B U}$, $D_{B U}, E_{T D}$, and $D_{T D}$}

Scalar entrainment and detrainment rates $E_{\mathrm{BU}}, D_{\mathrm{BU}}$, $E_{\mathrm{TD}}$, and $D_{\mathrm{TD}}$ determined from LES using (3.33) and (3.34) are shown in Figs. 5a,b. The breakdown of closure assumption (3.29) is evident from the large negative 

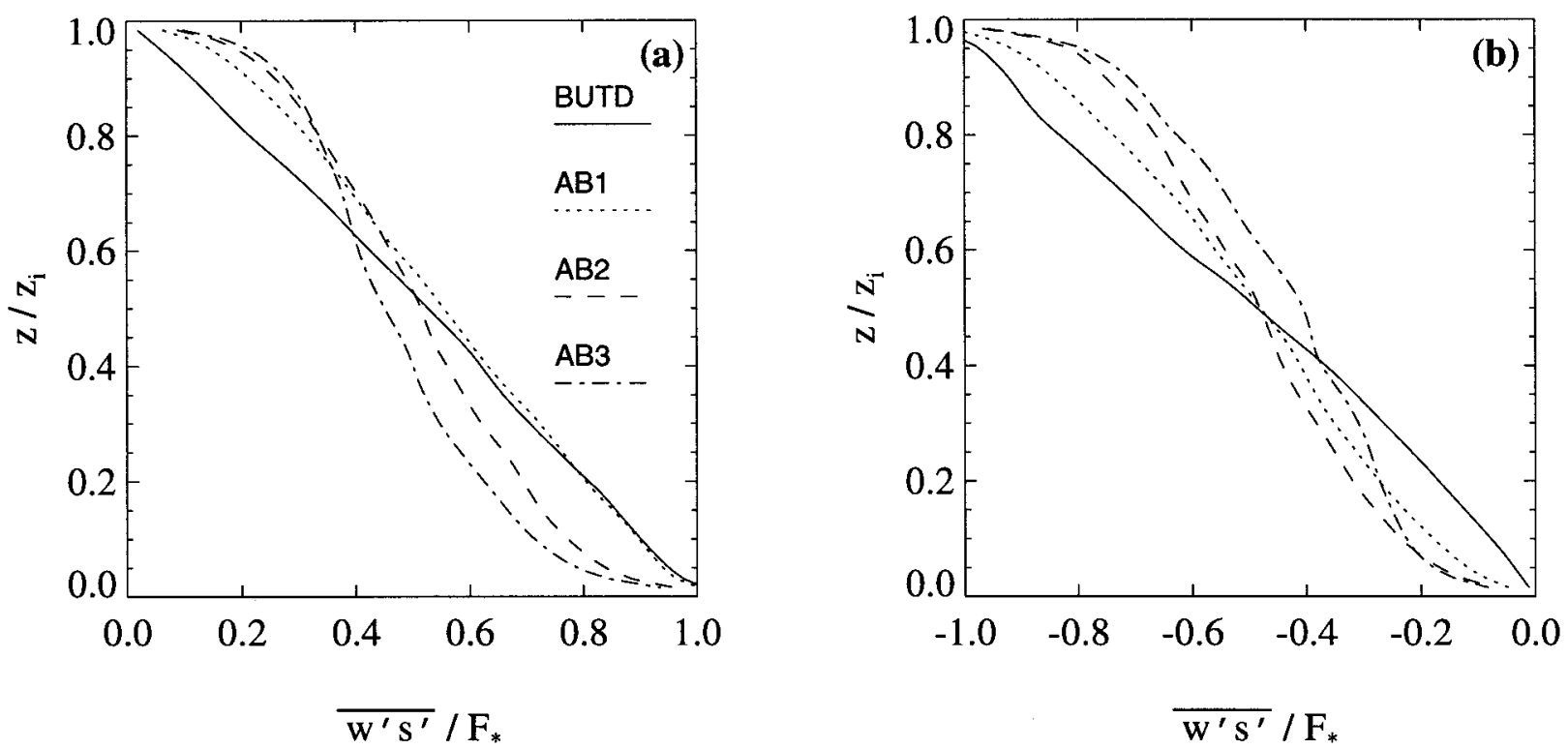

FIG. 2. Fluxes of (a) bottom-up diffusing scalars BU or A and (b) top-down diffusing scalars TD or B, determined from LES.

values of $E_{s}$ and $D_{s}$ near the respective zero-flux boundaries $\left(E_{\mathrm{BU}}\right.$ and $D_{\mathrm{BU}}$ near the top, and $E_{\mathrm{TD}}$ and $D_{\mathrm{TD}}$ near the bottom). Using these $E_{s}$ and $D_{s}$ profiles in a massflux scheme would give rise to unstable solutions. The cause of this problem is the fact that near the problematic boundaries the subinterface-scale lateral-exchange term ${\overline{v_{R}^{\prime} s^{\prime}}}^{i}$ gives rise to transport from the draft with the lower mean scalar value to the draft with the higher mean scalar value. This is possible due to the presence of strong countergradient fluxes near those boundaries and the presence of smaller eddies that are not repre- sented in the plume-budget equations. Since in the entraining CBL countergradient fluxes are only found for bottom-up diffusing scalars, this problem would in the real atmosphere only occur for purely bottom-up diffusing scalars and would be confined to the top part of the CBL.

As said, if we try to model this process with (3.29), using unchanged profiles of $E_{\mathrm{S}}$ and $D_{\mathrm{S}}$, we inevitably get an unstable mass-flux scheme. Since in practice we do not deal with purely bottom-up and top-down diffusing scalar fields we propose the following practical
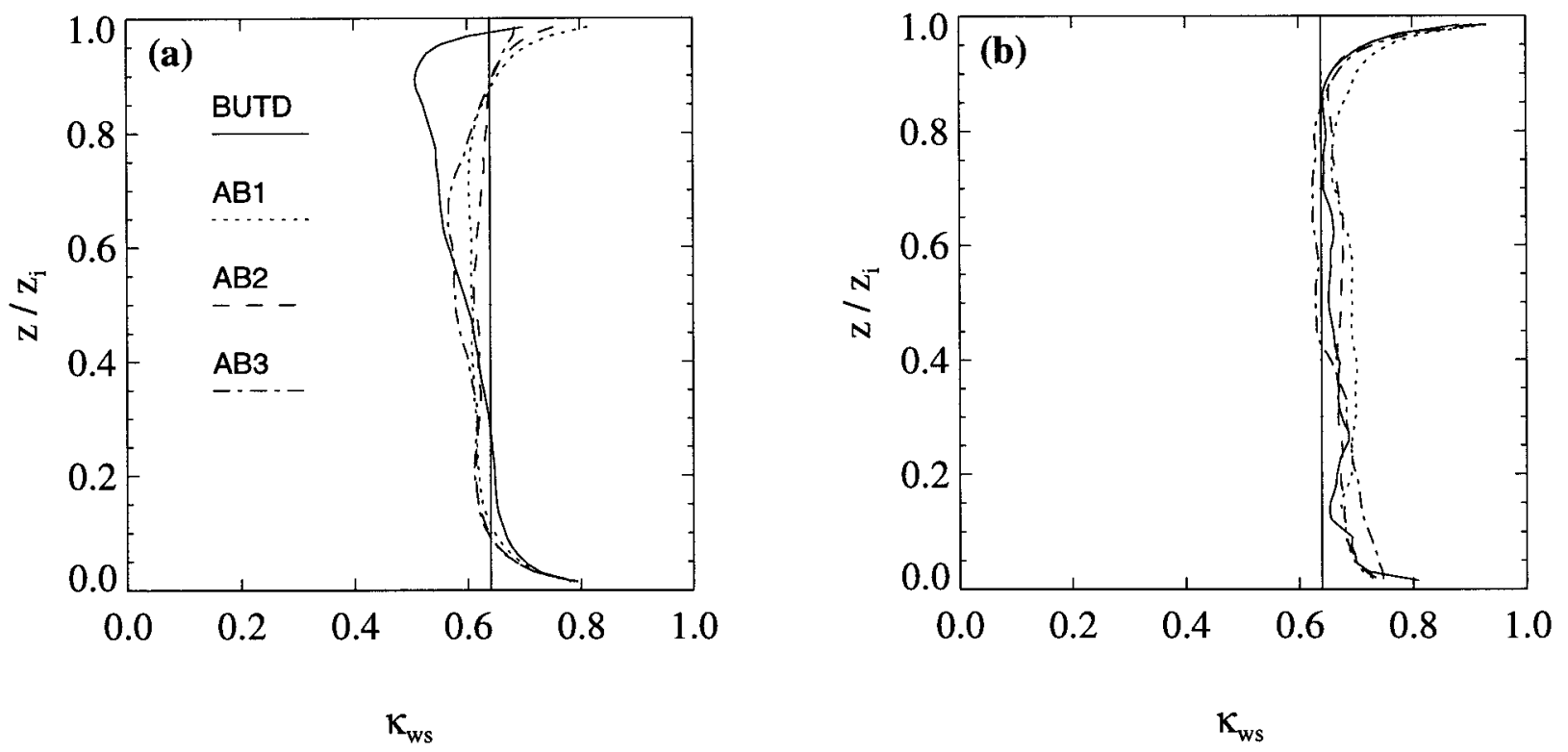

FIG. 3. Ratios of top-hat contribution to total flux for (a) bottom-up diffusing scalars BU or A and (b) top-down diffusing scalars TD or $\mathrm{B}$, determined from LES. The vertical lines indicate the theoretical value of 0.64 for a Gaussian joint pdf $P\left(w^{\prime}, s^{\prime}\right)$. 

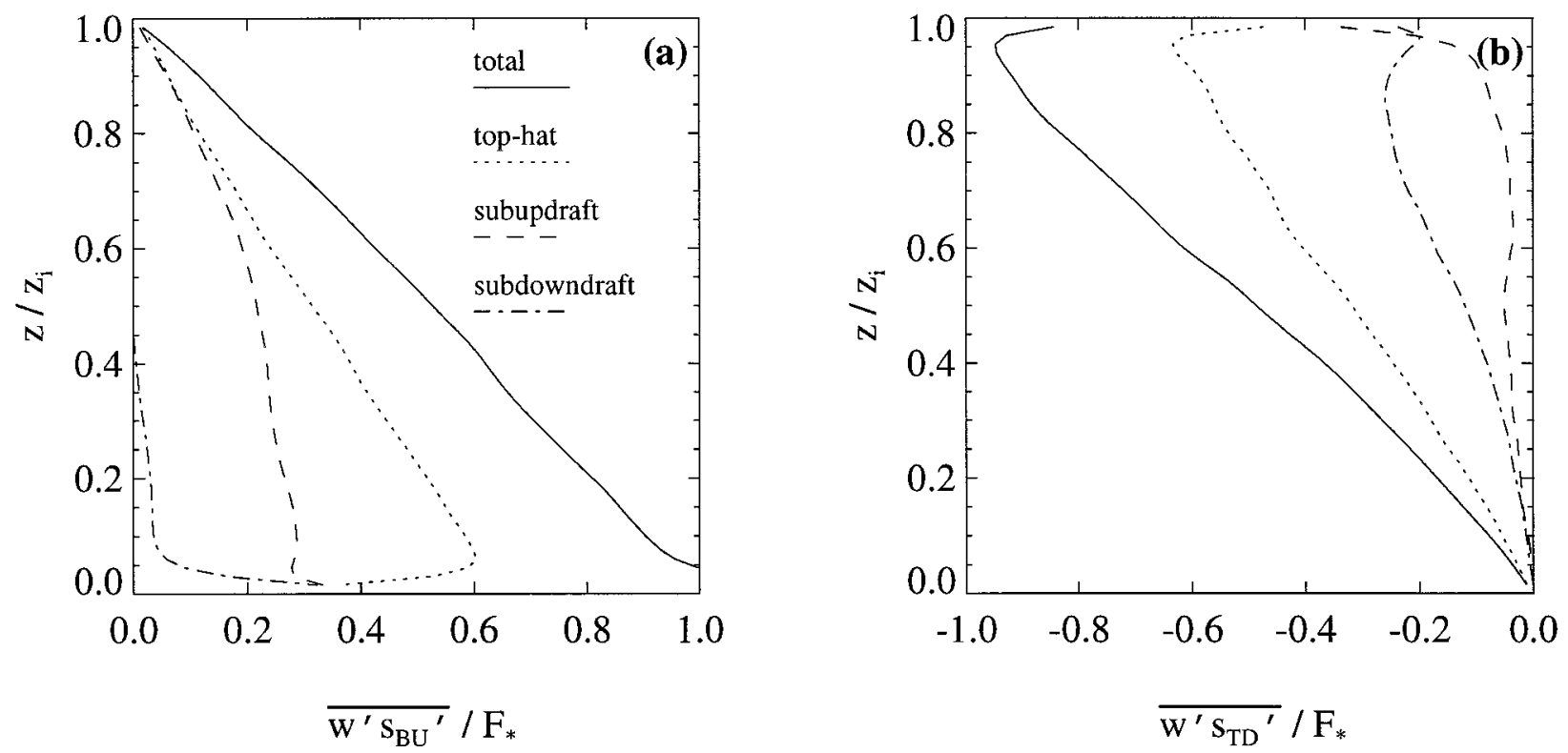

FIG. 4. Flux decomposition for (a) bottom-up diffusing scalar BU and (b) top-down diffusing scalar TD, determined from LES.

solution to the problem. We will ensure positivity by taking $E=\max \left(E_{\mathrm{BU}}, E_{\mathrm{TD}}\right)$ and $D=\max \left(D_{\mathrm{BU}}, D_{\mathrm{TD}}\right)$ and use these scalar-independent $E$ and $D$ instead of $E_{s}$ and $D_{s}$ in mass-flux scheme MF1.

\section{LES results for reactive scalars}

a. Steady-state scalar values $\overline{s_{A}}$ and $\overline{s_{B}}$

The steady-state scalar values for the reactive cases are listed as boundary layer averages in Table 2. As to be expected, the steady-state scalar values decrease with increasing reaction-rate coefficients. The variable that ultimately determines the steady-state concentrations is the covariance term $\overline{s_{\mathrm{A}}^{\prime} s_{\mathrm{B}}^{\prime}}$ in (2.15). A measure of the importance of this covariance term at each height is the intensity of segregation $I_{s}$, defined in (2.16) and plotted in Fig. 6. We find that $I_{s}$ becomes more negative for higher reaction rate coefficients, slowing down the horizontally averaged reaction rate by as much as $90 \%$ (compared to the horizontally well-mixed assumption) in case AB3. In Table 2 we have also given for each case the "bulk intensity of segregation," which repre-

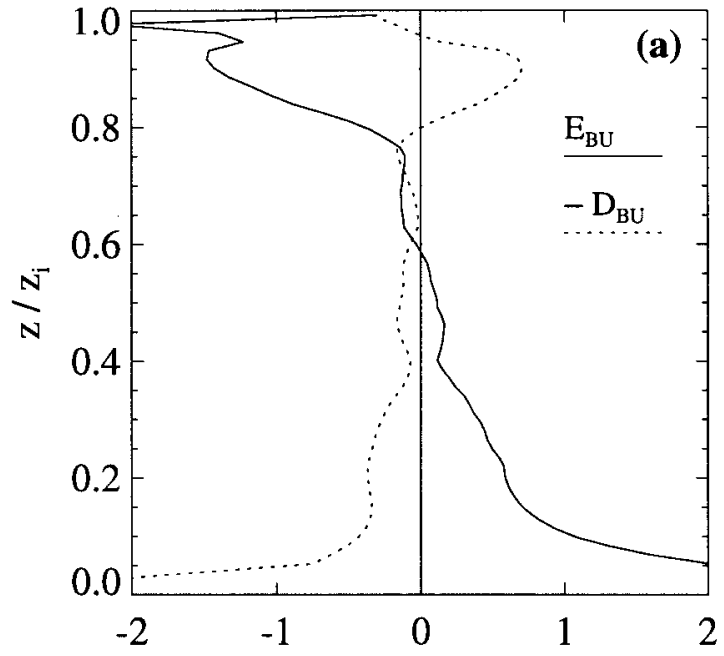

dimensionless units

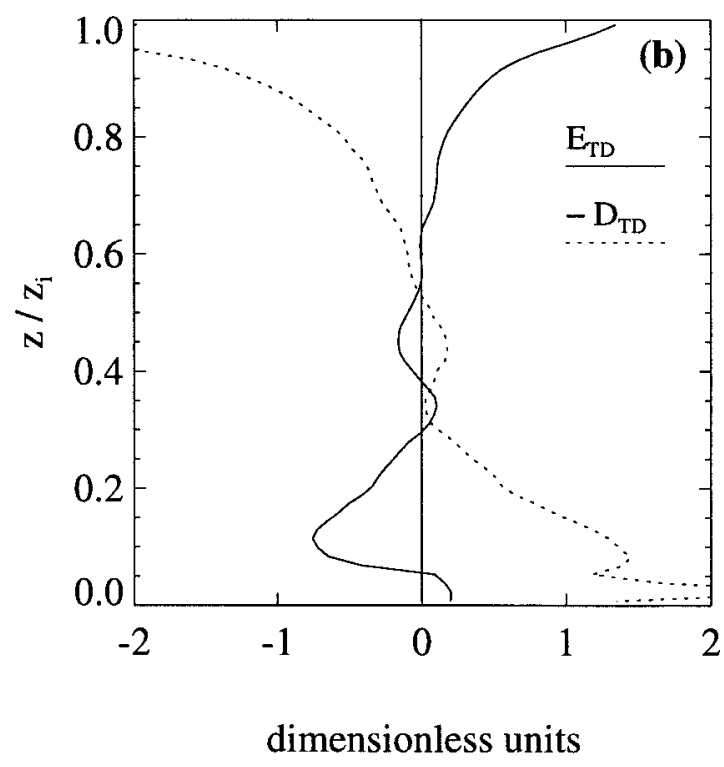

dimensionless units

FIG. 5. Entrainment and detrainment rates, determined from LES case BUTD for (a) bottom-up diffusing scalar BU and (b) top-down diffusing scalar TD. 
TABLE 2. Dimensionless reaction-rate coefficient (flux Damköhier number) $k$, and LES results for bulk quantities: mean steady-state concentrations $s_{\mathrm{A}}$ and $s_{\mathrm{B}}$, turbulent Damköhler number Da $\mathrm{a}_{\mathrm{t}}$, effective Damköhler number $\mathrm{Da}_{\text {eff }}$, bulk intensity of segregation $I_{s}$, and bulk ratio of top-hat contribution to total covariance.

\begin{tabular}{|c|c|c|c|c|c|c|}
\hline \multirow[b]{2}{*}{ Case } & \multirow[b]{2}{*}{$k$} & \multirow[b]{2}{*}{$s_{\mathrm{A}}, s_{\mathrm{B}}$} & \multirow[b]{2}{*}{$\mathrm{Da}_{\mathrm{t}}$} & \multirow[b]{2}{*}{$D a_{\text {eff }}$} & \multirow[b]{2}{*}{$I_{s}$} & $\kappa_{\mathrm{AB}} \overline{s_{\mathrm{A}}^{\prime} s_{\mathrm{B}}^{\prime}} d z$ \\
\hline & & & & & & $\overline{s_{\mathrm{A}}^{\prime} s_{\mathrm{B}}^{\prime}} d z$ \\
\hline AB1 & 0.20 & 2.8 & 0.55 & 0.13 & -0.34 & 0.18 \\
\hline AB2 & 1.0 & 1.8 & 1.8 & 0.32 & -0.68 & 0.22 \\
\hline AB3 & 5.0 & 1.4 & 7.2 & 0.49 & -0.90 & 0.23 \\
\hline $\mathrm{AB} \infty$ & $\infty$ & 1.4 & $\infty$ & 0.48 & -1 & 0.25 \\
\hline
\end{tabular}

sents the fractional change in bulk-averaged reaction rate (compared to the bulk well-mixed assumption). For the cases studied in this paper, the bulk value of $I_{s}$ is for the largest part determined by the horizontal segregation plotted in Fig. 6. In general, however, vertical segregation may also give an important contribution to the boundary layer averaged segregation. Concerning the steady-state concentrations listed in Table 2, we must be aware that the steady-state concentration of $1.4 s_{*}$ for scalars $A$ and $B$ in case $A B \infty$ is based on one instanteneous LES field only (as said before). Still we expect the real value to deviate not more than $0.05 s_{*}$ from this value, and we therefore conclude that the reaction-rate coefficient $k=5.0$ in case $\mathrm{AB} 3$ is already "close" to the infinite reaction-rate limit.

The boundary layer averaged scalar values for the reactive cases shown in Table 2 illustrate the limitation of chemical reaction due to incomplete mixing by convective turbulence (cf. Beets et al. 1996; Molemaker and Vilà-Guerau de Arellano 1998). We see that for increasing "turbulent Damköhler number" (defined as $\mathrm{Da}_{\mathrm{t}} \equiv k s_{\mathrm{A}} / s_{*}$ ), which is based on a well-mixed assumption, the effective Damköhler number, defined as $\mathrm{Da}_{\text {eff }} \equiv\left(1+I_{s}\right) \mathrm{Da}_{\mathrm{t}}$, reaches a finite limit. Thus it can be concluded that the convergence of $I_{s}$ to its limiting value of -1 exactly counteracts the increase of $\mathrm{Da}_{t}$ toward infinity. The steady-state concentrations reach a limiting value of $1.4 s_{*}$, corresponding to an effective Damköhler number of 0.5 . This limiting value is determined from a manipulation of the scalar fields of the nonreactive case, and no series of runs with increasing reaction-rate coefficients needs to be performed. Molemaker and Vilà-Guerau de Arellano (1998) using direct numerical simulation (DNS) of a CBL instead of LES did perform such a series of runs with their model. They found an asymptotic value for the effective Damköhler number of about $0.8,50 \%$ higher than our result. The source of this large difference probably lies in the much lower Rayleigh number Ra (and Reynolds number Re) used in DNS compared to LES. In our LES $\mathrm{Ra}$ and $\mathrm{Re}$ are several orders of magnitude larger than in their DNS and are close to real atmospheric values. Concerning the turbulent control of chemical reactions

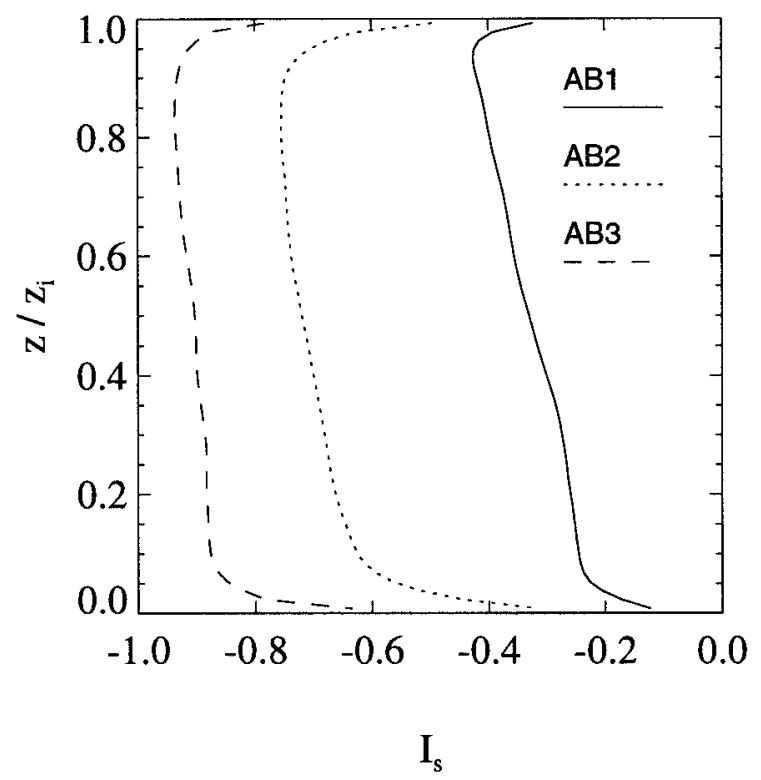

FIG. 6. Intensities of segregation for bottom-up and top-down diffusing scalars $\mathrm{A}$ and $\mathrm{B}$ in cases $\mathrm{AB} 1, \mathrm{AB} 2$, and $\mathrm{AB} 3$.

found here, one should be aware of the fact that we here study a special chemistry case with equal input fluxes and equal boundary layer averaged concentrations. A similar effect needs not be present in other chemistry cases.

\section{b. Fluxes $\overline{w^{\prime} s_{A}^{\prime}}$ and $\overline{w^{\prime} s_{B}^{\prime}}$}

In Figs. 2a,b also, the fluxes of the bottom-up and top-down scalars A and B in the reactive cases are plotted. The equilibrium between flux divergence and chemical destruction results in nonlinear flux profiles. The fact that the shape of the flux profiles of the top-down diffusing scalars differs somewhat from that of the bottom-up diffusing scalars indicates that in most of the cases we have not yet reached an exact steady state. However, since we found small tendencies for the boundary layer averaged concentrations, we consider the scalars to be close enough to steady state for the purposes of this paper. The nonlinearity of the flux profiles becomes stronger for higher reaction rates. The flux profiles for case $\mathrm{AB} \infty$ (not shown) are close to those of case AB3. Just as there exists a minimum limit on the concentrations there is a maximum limit, for the specific cases studied in this paper, on the nonlinearity of the flux profiles for higher and higher reaction rates. In other cases, for example with premixed emissions (positive covariances) or in cases where one of the species has a much higher initial concentration than the other species (for the sake of the argument keeping the fluxes fixed), the just-mentioned limits do not have to be present and the nonlinearity of the fluxes can be larger than in the cases studied in this paper. Preliminary results for other 
cases with larger flux divergences lead us to expect that the main results of this paper apply more generally.

\section{c. Covariance $\overline{s_{A}^{\prime} s_{B}^{\prime}}$}

Figures $7 \mathrm{a}, \mathrm{b}$ show profiles of the total covariance $\overline{s_{\mathrm{A}}^{\prime} s_{\mathrm{B}}^{\prime}}$ and the fractional top-hat contribution $\kappa_{\mathrm{AB}} \equiv$ $\frac{s_{\mathrm{A}}^{\prime} s_{\mathrm{B} \text { top-hat }}^{\prime}}{\overline{s_{\mathrm{A}}^{\prime} s_{\mathrm{B}}^{\prime}}}$, respectively. In the last column of Table 2 the boundary layer averaged ratios of top-hat contribution to total covariance (weighed with the covariance at each height) are listed. These boundary layer averaged quantities are the ones that should be modeled correctly by the mass-flux schemes in order to reach the correct boundary layer averaged steady-state concentrations of scalars A and B. The profile of the total covariance is not very sensitive to the change in reaction rate by a factor of 25 between cases $\mathrm{AB} 1$ and AB3: $\overline{s_{\mathrm{A}}^{\prime} s_{\mathrm{B}}^{\prime}}$ changes less than $50 \%$. And also $\kappa_{\mathrm{AB}}$ changes less than $50 \%$. Case $\mathrm{AB} 1$ has the lowest $\kappa_{\mathrm{AB}}$, indicating that subplume covariances become largest for $\mathrm{Da}_{\mathrm{t}}$ in the order of 1. For nonreacting scalars the covariance $\overline{s_{\mathrm{BU}}^{\prime} s_{\mathrm{TD}}^{\prime}}$ is smallest and the fractional contribution of the subplume covariances is somewhat smaller than for reactive scalars (not shown). In appendix A we show that $\kappa_{12}=0.25 \pm 0.10$ for all types of scalars in the solidlid CBL. In the parameterization for the subplume covariances (3.23) and (3.24) in schemes MF1 to MF3 we will use $\kappa_{\mathrm{AB}}=0.25$. On the basis of experiments in the entraining CBL Young (1988a) found values of $\kappa_{\theta \theta}$ (the fractional top-hat contribution to the total potential temperature variance) in the surface layer and lower mixed layer that lie within the stated range. Also LES results for a case in the entraining CBL (not shown; this case is also based on simulating a steady state in the CBL with equal bulk quantities of reactive bottom-up and top-down diffusing scalars) confirm that one can extrapolate the result to the surface layer and lower mixed layer of the entraining CBL. The top-hat formula for the covariance, however, breaks down for reactions that mainly take place in the entrainment zone. We anticipate that such conditions do not often occur in reality. In future studies we will assess the importance of this problem for realistic cases in atmospheric chemistry.

In order to assess the assumption made in (3.23) and (3.24) that the subplume covariances are equal for updrafts and downdrafts, we have plotted in Fig. 8 the terms of the covariance decomposition given in (3.22). Only one case (AB2) is shown; the other cases give similar results. Apparently the subupdraft and subdowndraft contributions are not equal at each height, as assumed in our subplume covariances parameterization, but compared to the large differences found for the subplume contributions to the fluxes, the subplume contributions to the covariance are relatively close to each other in size. We expect that the differences do not have a large influence on the performance of the mass-flux schemes, since the chemical sinks and sources are relatively small terms in (3.6) and (3.7) (not shown) and are only important in the boundary layer averaged budget, in which they balance the input terms from the boundary fluxes.

Complementary to our updraft-downdraft analyses of $\overline{s_{\mathrm{A}}^{\prime} s_{\mathrm{B}}^{\prime}}$, we have also investigated the behavior of $\overline{s_{\mathrm{A}}^{\prime} s_{\mathrm{B}}^{\prime}}$ for the different cases in spectral space (see appendix B).

\section{d. Lateral entrainment and detrainment rates $E_{A}, D_{A}$, $E_{B}$, and $D_{B}$}

For the profiles of $E_{\mathrm{A}}, D_{\mathrm{A}}, E_{\mathrm{B}}$, and $D_{\mathrm{B}}$ (not shown) we find similar results as for $E_{\mathrm{BU}}, D_{\mathrm{BU}}, E_{\mathrm{TD}}$, and $D_{\mathrm{TD}}$, respectively (shown in Figs. 5a,b). The only difference is that near the zero-flux boundaries the profiles do not become negative but only become zero. This is due to the fact that the large chemical sink term near both boundaries prevents strong countergradient fluxes from occurring (see Figs. 10a,b for typical LES profiles of scalars $\mathrm{A}$ and $\mathrm{B}$, respectively). Nevertheless, we will also use $E=\max \left(E_{\mathrm{BU}}, E_{\mathrm{TD}}\right)$ and $D=\max \left(D_{\mathrm{BU}}, D_{\mathrm{TD}}\right)$ in scheme MF1 for the reactive cases.

\section{Results of mass-flux schemes}

\section{a. Nonreactive case BUTD}

The quasi-steady results of four mass-flux schemes are shown in Figs. 9a,b (for this case scheme MF5 is identical to scheme MF3). The LES results are also plotted. The effect of using the new subplume-fluxes parameterization (3.14) and (3.15) is a change in the gradient in the bulk of the CBL to a (stronger) countergradient profile. The two schemes containing the new subplume-fluxes parameterization (schemes MF1 and MF2) model gradients in the bulk of the CBL that are closer to the LES results than the modeled gradients by the other two schemes.

Adding subinterface-scale lateral exchange (scheme MF1 compared to scheme MF2) results in larger scalar values near the influx boundaries of the scalars (more in agreement with LES) and a corresponding decrease of scalar values in the bulk of the CBL. The behavior near the influx boundaries is comparable for three of the schemes (MF1, MF3, and MF4) and is closer to LES for these schemes than for scheme MF2.

The discrepancies between all mass-flux schemes and LES near the zero-flux boundaries are due to the two different closure assumptions studied, (3.26) and (3.29). However, as said before, we can argue that for realistic scalar fields consisting of both bottom-up and top-down parts, the problems near the zero-flux boundaries for the purely bottom-up and top-down diffusing scalars are mitigated.

Although none of the schemes matches the LES results, we can conclude that the most comprehensive scheme (MF1) that is closest to the exact plume-budget equations gives the best overall performance of the schemes considered. 

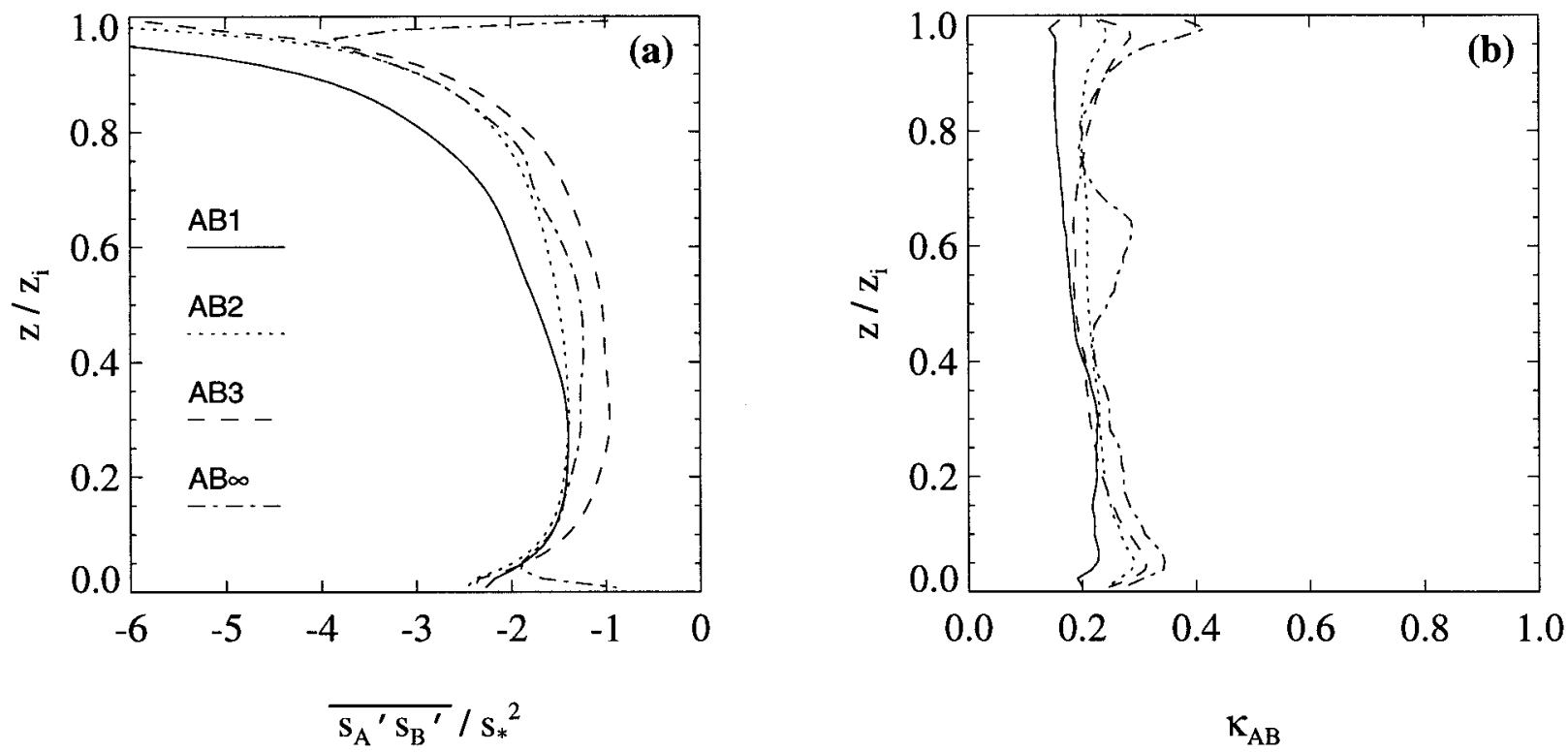

FIG. 7. (a) Total covariances of reactive bottom-up and top-down diffusing scalars A and B and (b) ratios of top-hat contribution to total covariance, determined from LES.

\section{b. Reactive cases $A B 1, A B 2$, and $A B 3$}

In Table 3 the boundary layer averaged steady-state results for the reactive cases are listed for all five massflux schemes. We have added the results for a simple bulk boundary layer scheme without covariance parameterization, which is also indicative for the results of nonlocal scalar transport schemes without (implicit) covariance parameterization. From Table 3 it becomes clear that schemes

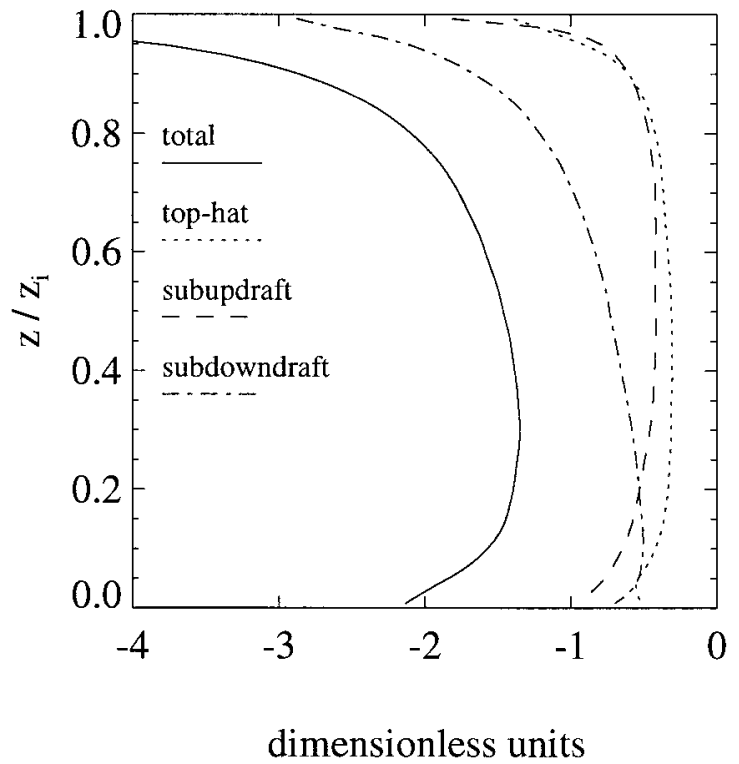

FIG. 8. Covariance decomposition for bottom-up and top-down diffusing scalars A and B in case AB2, determined from LES. Shown are total resolved covariance, top-hat contribution, subupdraft contribution, and subdowndraft contribution.
MF1 through MF3 have a similar good performance for the boundary layer averaged results. These are the three mass-flux schemes that include the subplume-covariances parameterization (3.23) and (3.24). Scheme MF4 performs worse but still gives a much improved performance compared to the bulk scheme, due to the fact that the updraftdowndraft scalar difference $\left(\bar{s}^{u}-\bar{s}^{d}\right)$ is erroneously overestimated by a factor $\kappa_{w s}^{-1}$. Finally, scheme MF5 does not give much improvement in performance compared to the bulk scheme.

For case AB2, Figs. 10a,b show the scalar profiles modeled by the five mass-flux schemes in comparison to the LES results. As in the nonreactive case BUTD the profiles modeled by scheme MF1 are closest to the LES profiles near the influx boundaries. And, also as in case BUTD, the effect of including subplume fluxes explicitly is a significant change of the gradient in the bulk of the CBL.

The subplume-covariances parameterization is the determining factor for the performance of the mass-flux schemes in the reactive cases. Therefore we have plotted in Fig. 11 the profiles of the intensity of segregation $I_{s}$ for case AB2. Compared to the profiles of $I_{s}$ modeled by schemes MF4 and MF5, the profiles modeled by schemes MF1, MF2, and MF3 are relatively close to LES.

Due to the fact that the subplume-covariances parameterization has the largest impact on the boundary layer averaged steady-state concentrations, the other differences between the mass-flux schemes are irrelevant from the bulk point of view. However, in the studied cases we have prescribed the fluxes at the bottom and top. For a correct interactive modeling of the emission (deposition) at the surface and scalar entrainment (detrainment) fluxes at the top it is also important to correctly model scalar profiles near the bottom and top boundaries of the CBL. 

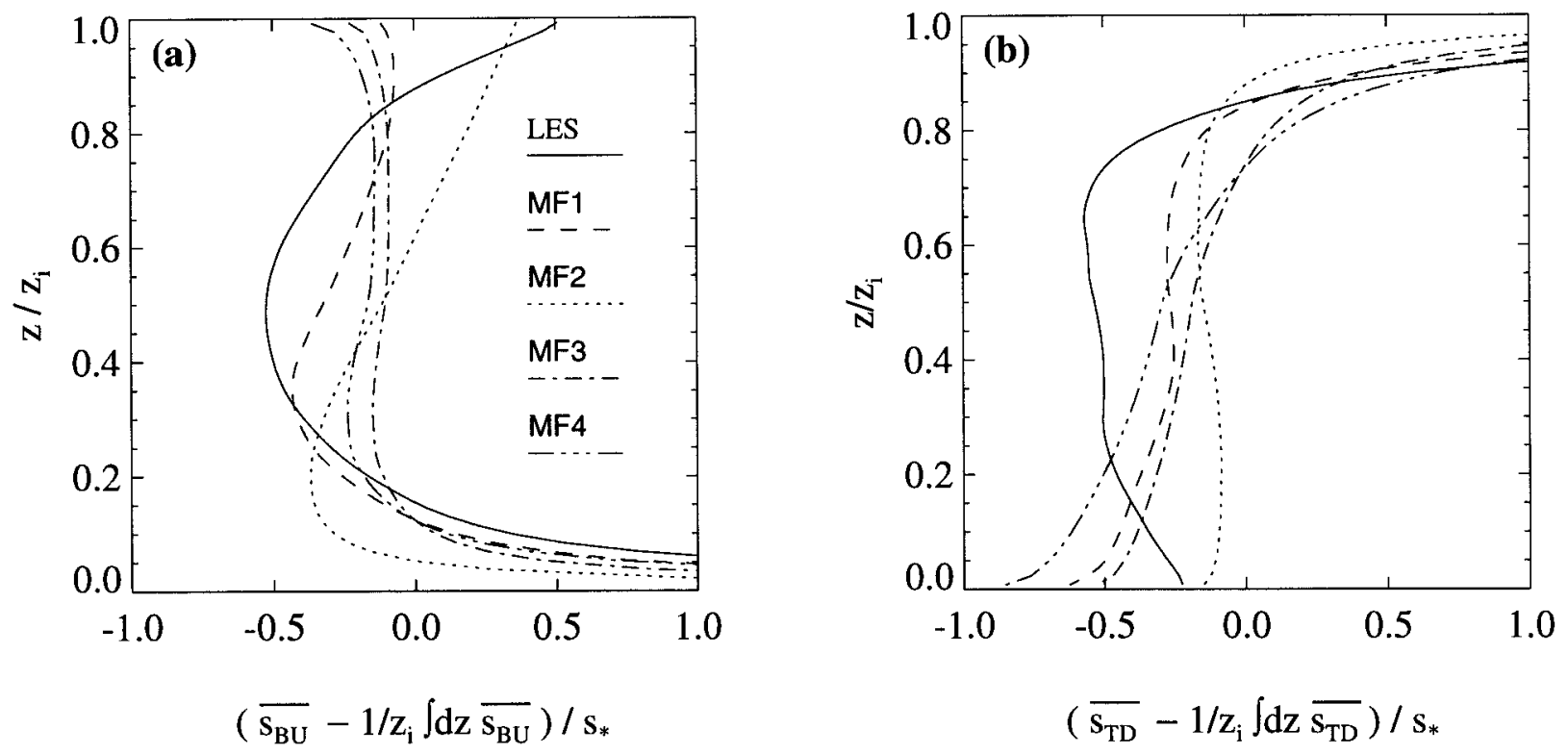

FIG. 9. Mean quasi-steady profiles of (a) bottom-up diffusing scalar BU and (b) top-down diffusing scalar TD in nonreactive case BUTD, modeled by four mass-flux schemes and compared to LES. The steady deviation from the bulk concentration is plotted.

\section{Summary and discussion}

In this paper we have studied the mass-flux characteristics of scalar transport in the CBL. We have explicitly evaluated different parameterizations for component processes that can be included in mass-flux schemes for transport of nonreactive and reactive scalars. The best performance is obtained with the mass-flux scheme that is nearly identical to the exact scalar plume-budget equations.

We have shown that a subplume-fluxes parameterization based on the different behaviors of bottom-up and top-down diffusing scalars is best able to model the gradients of bottom-up and top-down diffusing scalars in the bulk of the CBL. Furthermore, we have shown that for an adequate modelling of the lateral-exchange processes between plumes one also has to take into account the subinterface-scale lateral-exchange processes. Massflux scheme MF3 gives a satisfactory performance in modeling the boundary layer averaged concentrations of reactive scalars, the slightly more sophisticated scheme MF2 performs worse, and the most sophisticated scheme MF1 gives the best performance. However, we think that the improvement gained in scheme MF1 is not worth the increase in complexity. We advise using scheme MF3, in which the lateral-exchange terms are parameterized according to (3.26)-(3.28) and the subplume fluxes are parameterized according to (3.10) and (3.11).

For reactive scalars involved in a binary reaction (either as a reactant or as a reaction product) with moderate or fast reaction rates relative to the convective timescale, mass-flux schemes offer the advantage of intrinsically modeling the covariances of reactants. This gives a measure of the intensity of segregation of the scalars, which can give important corrections to the mean reaction rate. The covariance is dominated by the subplume contribution (75\% of the total covariance is caused by subplume covariances and the remaining $25 \%$ is represented by the top-hat approximation). We have used this result in three of the five mass-flux schemes studied and it turned out to be the determining component process parameterization for correctly modeling the boundary layer averaged steady-state concentrations. In typical reaction schemes for atmospheric chemistry many important reactions are moderately fast or fast compared to the convective timescale (like the $\mathrm{NO}+\mathrm{O}_{3}$ and $\mathrm{C}_{5} \mathrm{H}_{8}+\mathrm{OH}$ reactions). However, at present it is not clear whether or not the segregation of chemical species due to convection is an important effect that has to be parameterized in large-scale atmospheric chemistry models. The use of the top-hat approximation for the covariance and the param-

TABLE 3. Bulk mean steady-state concentrations of scalars A and B (these are equal), determined from LES and modeled by five massflux schemes. In brackets the relative deviation from the LES value is given. For comparison we have also included the results of a bulk boundary layer scheme without covariance parametrization (BULK).

\begin{tabular}{cccccccc}
\hline \hline Case & LES & BULK & MF1 & MF2 & MF3 & MF4 & MF5 \\
\hline AB1 & 2.76 & $2.24(-19 \%)$ & $2.56(-7.2 \%)$ & $2.51(-9.1 \%)$ & $2.54(-8.0 \%)$ & $2.46(-11 \%)$ & $2.32(-16 \%)$ \\
AB2 & 1.78 & $1.00(-44 \%)$ & $1.68(-5.6 \%)$ & $1.62(-9.0 \%)$ & $1.64(-7.9 \%)$ & $1.49(-16 \%)$ & $1.21(-32 \%)$ \\
AB3 & 1.43 & $0.45(-69 \%)$ & $1.48(+3.5 \%)$ & $1.41(-1.4 \%)$ & $1.39(-2.8 \%)$ & $1.24(-13 \%)$ & $0.86(-40 \%)$ \\
\hline
\end{tabular}




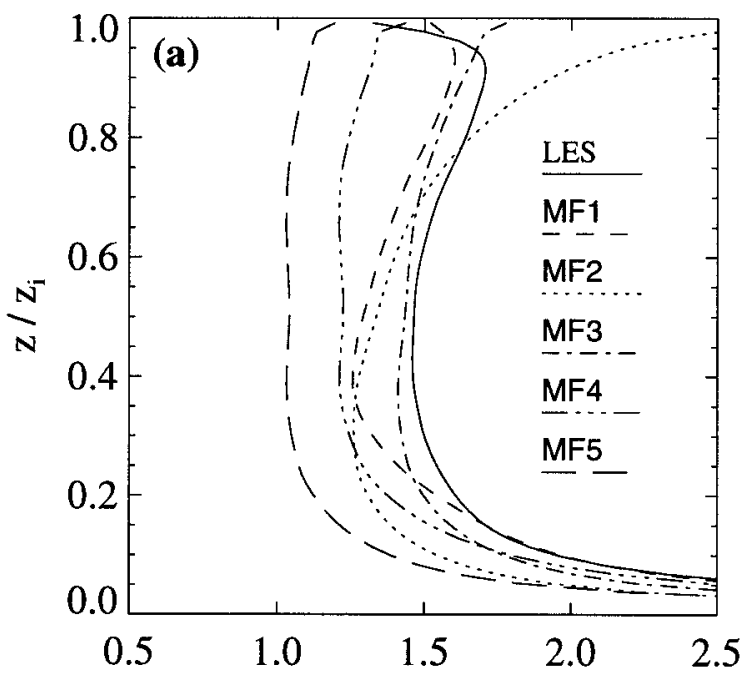

$\overline{\mathrm{s}_{\mathrm{A}}} / \mathrm{s}_{*}$

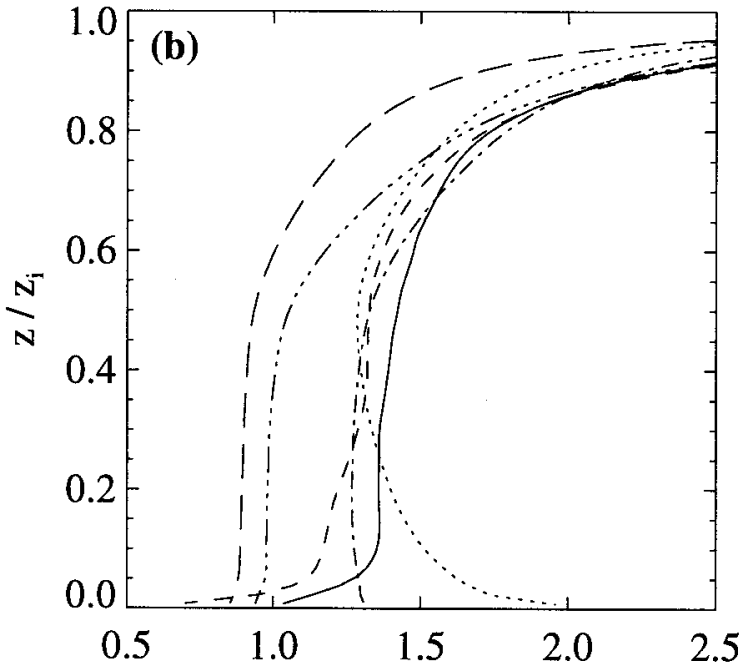

$\overline{\mathrm{s}_{\mathrm{B}}} / \mathrm{s}_{*}$

FIG. 10. Mean steady-state profiles of (a) bottom-up diffusing scalar A and (b) top-down diffusing scalar B in reactive case AB2, modeled by five mass-flux schemes and compared to LES.

eterization for the subplume covariances that we propose here, can help in assessing the importance of the effect. The uncertainty of about $40 \%$ in the value of $\kappa_{\mathrm{AB}}$ (the fractional top-hat contribution to the total covariance) is acceptable in this light. The primary concern is to model more accurate effective reaction rates in large-scale atmospheric chemistry-transport models than is currently the case. The effective reaction rates modeled with massflux schemes that include the subplume covariances pa-

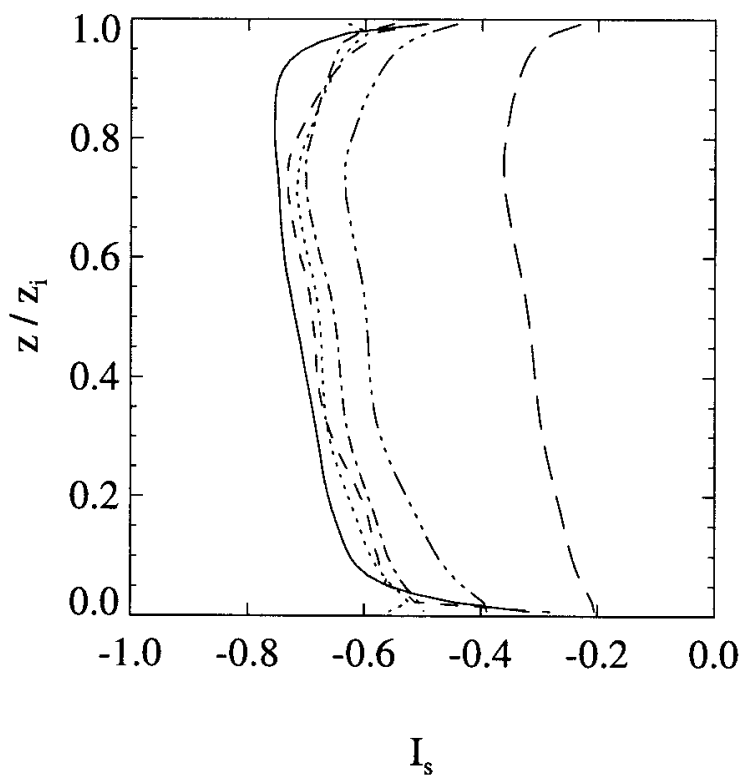

FIG. 11. Intensity of segregation for bottom-up and top-down diffusing scalars $\mathrm{A}$ and $\mathrm{B}$ in case $\mathrm{AB} 2$, modeled by five mass-flux schemes and compared to LES. The line styles are as in Fig. 10. rameterization are more accurate than the mean reaction rates calculated without this parameterization, even with the large uncertainty in $\kappa_{\mathrm{AB}}$.

One must be aware that one cannot directly include the mass-flux scheme MF3 in large-scale atmospheric models. In this paper we have prescribed the profiles related to boundary layer dynamics that are needed to drive the mass-flux schemes (i.e., the mass flux and the updraft area fraction, or, equivalently, the second and third moments of the turbulent vertical velocity; see appendix C). In practice not all of these dynamical quantities are available in large-scale atmospheric models, and it must be recognized that it is not yet clear whether adding them to the models will lead to a scalar transport scheme that is at least as accurate and robust as the schemes that are currently used for scalar transport in the CBL. In future studies we will address this issue.

Acknowledgments. Arthur Petersen acknowledges the facilities offered by the Royal Netherlands Meteorological Institute (KNMI), partner in the Netherlands Centre for Climate Research (CKO) during a one and a half year stay at KNMI. Harm Jonker calculated the spectra. Useful comments on previous versions of the manuscript were received from Peter Bechtold, Bert Holtslag, Aad van Ulden, Gé Verver, and two anonymous reviewers. Arthur Petersen and Kees Beets acknowledge support by the Netherlands Geosciences Foundation (GOA) with financial aid from the Netherlands Organization for Scientific Research (NWO). This work was sponsored by the National Computing Facilities Foundation (NCF) for the use of supercomputing facilities. 


\section{APPENDIX A}

\section{Fractional Top-Hat Contribution to the Covariance $\kappa_{12}$}

In this appendix we want to generalize the work of Wyngaard and Moeng (1992) from fluxes to variances and covariances. Wyngaard and Moeng (1992) determined a theoretical value of $\kappa_{w s}$, assuming a Gaussian probability density function (pdf) $P\left(w^{\prime}, s^{\prime}\right.$ ). We here determine an expression for $\kappa_{12}$, the top-hat fraction of the covariance of two (arbitrary) scalars, assuming Gaussian pdf's $P_{1}\left(w^{\prime}, s_{1}^{\prime}\right)$ and $P_{2}\left(w^{\prime}, s_{2}^{\prime}\right)$. Furthermore, we would like to demonstrate that $\kappa_{12}=0.25$ is a bestguess value for all possible scalars, thereby generalizing from the pure bottom-up and top-down diffusing scalar fields studied in this paper. Finally, this appendix aims to determine the uncertainty in this value of $\kappa_{12}$.

We start with the definition of $\kappa_{12}$ :

$$
\kappa_{12} \equiv \frac{a{\overline{s_{1}}}^{u}{\overline{S_{2}}}^{u}+(1-a){\overline{s_{1}}}^{d}{\overline{s_{2}}}^{d}-\overline{s_{1}} \overline{s_{2}}}{\overline{s_{1}^{\prime} s_{2}^{\prime}}} .
$$

We rewrite the updraft and downdraft quantities in terms of the joint pdf $P\left(w^{\prime}, s^{\prime}\right)$ of vertical velocity and scalar fluctuations, analogous to Wyngaard and Moeng (1992):

$$
\begin{gathered}
{\overline{\bar{s}_{1}}}^{u} \equiv \frac{\int_{-\overline{s_{1}}}^{\infty} \int_{0}^{\infty} s_{1}^{\prime} P\left(w^{\prime}, s_{1}^{\prime}\right) d w^{\prime} d s_{1}^{\prime}}{\int_{-\overline{s_{1}}}^{\infty} \int_{0}^{\infty} P\left(w^{\prime}, s_{1}^{\prime}\right) d w^{\prime} d s_{1}^{\prime}}, \text { and } \\
{\overline{s_{1}}}^{d} \equiv \frac{\int_{-\overline{s_{1}}}^{\infty} \int_{-\infty}^{0} s_{1}^{\prime} P\left(w^{\prime}, s_{1}^{\prime}\right) d w^{\prime} d s_{1}^{\prime}}{\int_{-\overline{s_{1}}}^{\infty} \int_{-\infty}^{0} P\left(w^{\prime}, s_{1}^{\prime}\right) d w^{\prime} d s_{1}^{\prime}},
\end{gathered}
$$

with similar expressions for $s_{2}^{u}$ and $s_{2}^{d}$. The lower integration limit $-\overline{s_{1}}$ for $s_{1}^{\prime}$ follows from the fact that the scalar values cannot become negative $\left(\overline{s_{1}}\right.$ is the horizontally averaged value of scalar 1 relative to which the fluctuations $s_{1}^{\prime}$ are defined).

We define the correlation coefficients $\rho$ in the following way:

$$
\begin{aligned}
& \overline{w^{\prime} s_{1}^{\prime}} \equiv \rho_{w s_{1}} \sigma_{w} \sigma_{s_{1}}, \\
& \overline{w^{\prime} s_{2}^{\prime}} \equiv \rho_{w s_{2}} \sigma_{w} \sigma_{s_{2}}, \quad \text { and } \\
& \overline{s_{1}^{\prime} s_{2}^{\prime}} \equiv \rho_{s_{1} s_{2}} \sigma_{s_{1}} \sigma_{s_{2}},
\end{aligned}
$$

where the $\sigma$ 's denote the rms values of the indicated quantities.

If $P\left(w^{\prime}, s_{1}^{\prime}\right)$ and $P\left(w^{\prime}, s_{2}^{\prime}\right)$ have a Gaussian form, it follows that

$$
\begin{aligned}
& {\overline{s_{1}}}^{u}=\frac{2 \sigma_{s_{1}} \rho_{w s_{1}}}{\sqrt{2 \pi}}, \text { and } \\
& {\overline{s_{1}}}^{d}=\frac{2 \sigma_{s_{s}} \rho_{w s_{1}}}{\sqrt{2 \pi}},
\end{aligned}
$$

and similar expressions are obtained for scalar $s_{2}$. Furthermore, it follows under Gaussian assumptions that $a$ $=\frac{1}{2}$. Combining all above equations we arrive at

$$
\kappa_{12, \mathrm{Gauss}}=\frac{4}{2 \pi} \frac{\rho_{w s_{1}} \rho_{w s_{2}}}{\rho_{s_{1} s_{2}}} .
$$

Contrary to what was found earlier for $\kappa_{w s}$ (the top-hat

\begin{tabular}{|c|c|c|c|c|c|c|}
\hline \multirow[b]{2}{*}{ Description } & \multirow[b]{2}{*}{$\alpha_{1}$} & \multirow[b]{2}{*}{$\beta_{1}$} & \multirow[b]{2}{*}{$\alpha_{2}$} & \multirow[b]{2}{*}{$\beta_{2}$} & \multirow{2}{*}{$\frac{\kappa_{12, \text { Gauss }} \overline{s_{1}^{\prime} s_{2}^{\prime}} d z}{\int \overline{s_{1}^{\prime} s_{2}^{\prime}} d z}$} & \multirow{2}{*}{$\frac{\kappa_{12} \overline{s_{1}^{\prime} s_{2}^{\prime}} d}{\int \overline{s_{1}^{\prime} s_{2}^{\prime}} d z}$} \\
\hline & & & & & & \\
\hline $\begin{array}{l}s_{1}: \text { Pure bottom-up } \\
s_{2}: \text { Pure top-down }\end{array}$ & 1.0 & 0.0 & 0.0 & 1.0 & 0.41 & 0.36 \\
\hline $\begin{array}{l}s_{1}: 20 \% \text { detrained at top } \\
s_{2}: \text { No deposition at bottom }\end{array}$ & 1.0 & -0.2 & 0.0 & 1.0 & 0.17 & 0.16 \\
\hline $\begin{array}{l}s_{1}: 20 \% \text { detrained at top } \\
s_{2}: 20 \% \text { deposited at bottom }\end{array}$ & 1.0 & -0.2 & -0.2 & 1.0 & 0.19 & 0.18 \\
\hline $\begin{array}{l}s_{1}: 20 \% \text { detrained at top } \\
s_{2}: 100 \% \text { deposited at bottom }\end{array}$ & 1.0 & -0.2 & -1.0 & 1.0 & 0.23 & 0.22 \\
\hline Both pure bottom-up & 1.0 & 0.0 & 1.0 & 0.0 & 0.28 & 0.29 \\
\hline $\begin{array}{l}\text { Both bottom-up } \\
20 \% \text { detrained at top }\end{array}$ & 1.0 & -0.2 & 1.0 & -0.2 & 0.27 & 0.27 \\
\hline $\begin{array}{l}s_{1}: 20 \% \text { entrained at top } \\
s_{2}: 20 \% \text { detrained at top }\end{array}$ & 1.0 & 0.2 & 1.0 & -0.2 & 0.32 & 0.33 \\
\hline Both pure top-down & 0.0 & 1.0 & 0.0 & 1.0 & 0.07 & 0.09 \\
\hline
\end{tabular}
flux fraction) in the case of a Gaussian joint pdf $P\left(w^{\prime}\right.$, $\left.s^{\prime}\right)$, that is, that $\kappa_{w s}=4 /(2 \pi)$ is independent of the correlation coefficient $\rho_{w s}$, Eq. (A.9) states that $\kappa_{12}$ is dependent on all three correlation coefficients defined in (A.4)-(A.6). We can also see that if we substitute $w$ for either $s_{1}$ or $s_{2}$, the result $\kappa_{w s}=4 /(2 \pi)$ for the top-

TABLE A1. Bulk ratios of top-hat contribution to total covariance for different combinations of nonreactive bottom-up and top-down scalar fields determined from LES. 
hat flux fraction comes out again. If we assume $s_{1}=s_{2}$ $\equiv s$ we find for the top-hat variance fraction $\kappa_{s s, \text { Gauss }}=$ $4 /(2 \pi) \rho_{w s}^{2}$.

In order to empirically investigate the sensitivity of $\kappa_{12}$ to different cases we have used the superposition hypothesis of Wyngaard and Brost (1984) to construct different types of scalars $s_{1}$ and $s_{2}$ (without chemical reaction) from the $\mathrm{BU}$ and $\mathrm{TD}$ scalars in the following manner:

$$
\begin{aligned}
& s_{1}=\alpha_{1} s_{\mathrm{BU}}+\beta_{1} s_{\mathrm{TD}}, \quad \text { and } \\
& s_{2}=\alpha_{2} s_{\mathrm{BU}}+\beta_{2} s_{\mathrm{TD}} .
\end{aligned}
$$

We do not have to perform additional LES runs in order to calculate $\kappa_{12}$ and $\kappa_{12, \text { Gauss }}$. In Table A1 we present the bulk values of $\kappa_{12}$ and $\kappa_{12 \text {,Gauss }}$ for a host of different cases, together spanning a large range of posibilities. We can conclude that in general the bulk value of $\kappa_{12 \text {,Gauss }}$ does not deviate much from the bulk value of $\kappa_{12}$. Furthermore, $\kappa_{12}=0.25 \pm 0.10$ seems to be a good estimate of the range of values that $\kappa_{12}$ can attain. From the experimental LES results presented in section 5 we infer that chemical reactions cannot cause large changes in this range.

\section{APPENDIX B}

\section{Spectral Behavior of $\overline{s_{1}^{\prime} s_{2}^{\prime}}$}

In this appendix we describe the spectral behavior of the covariance. The bottom-up scalars BU and A are denoted by $s_{1}$ and the top-down scalars TD and B by $s_{2}$. Using the $2 \mathrm{D}$ Fourier transforms $\widehat{s_{1}}$ and $\widehat{s_{2}}$, normalized in a proper way, we have

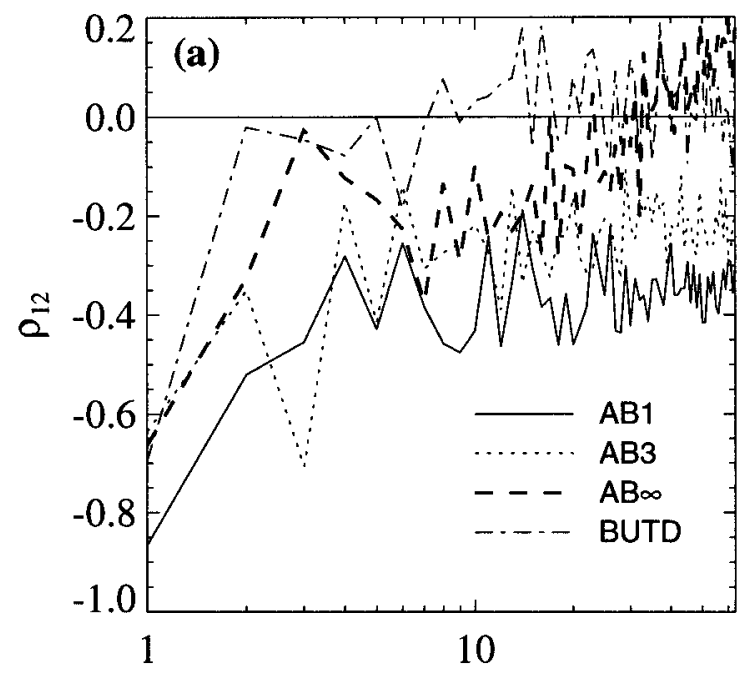

$\mathrm{k}$

$$
\overline{s_{1}^{\prime} s_{2}^{\prime}}=\sum_{i=-N}^{N} \sum_{j=-N}^{N}\left({\widehat{s_{1}}}^{i j}\right)^{*}{\widehat{s_{2}}}^{i j}=\sum_{k=1}^{N} S_{12}^{k},
$$

where $S_{12}$ is the discrete cospectrum of $s_{1}$ and $s_{2}$ (for the resolution of $130 \times 130$ we have $N=63$ ). Another spectral quantity that we investigate is the spectral correlation, defined as

$$
\rho_{12}^{k}=\frac{S_{12}^{k}}{\sqrt{S_{11}^{k} S_{22}^{k}}}
$$

where $S_{11}$ and $S_{22}$ are the discrete spectra of $s_{1}$ and $s_{2}$, respectively. The spectral correlation obeys $\rho_{12}^{k} \in[-1$, $1], k=1, \ldots, N$.

In Figs. B1a,b we have plotted $\rho_{12}$ and $S_{12}$ for different cases at heights $z / z_{i}=0.14$. The spectral behavior at other height is qualitatively similar (not shown). We see that case $\mathrm{AB} \infty$ behaves as case BUTD: the correlation drops to near-zero values for higher wavenumbers (smaller scales), while the reactive cases AB1 and AB3 (and $\mathrm{AB} 2$, not shown) maintain constant correlations as low as -0.35 at all scales for case AB1. This difference is due to the chemical reaction that proceeds at a moderate reaction rate in case $\mathrm{AB} 1$, acting at all scales as a source of negative covariance and counteracting the tendency toward near-zero correlations (which is dominant at the smaller scales for nonreactive or fast-reactive cases). From the cospectra $S_{12}$ we can furthermore conclude that the small scales have a larger contribution to the total covariance in case $\mathrm{AB} 1$ with a moderate reaction-rate coefficient than in the nonreactive case BUTD.

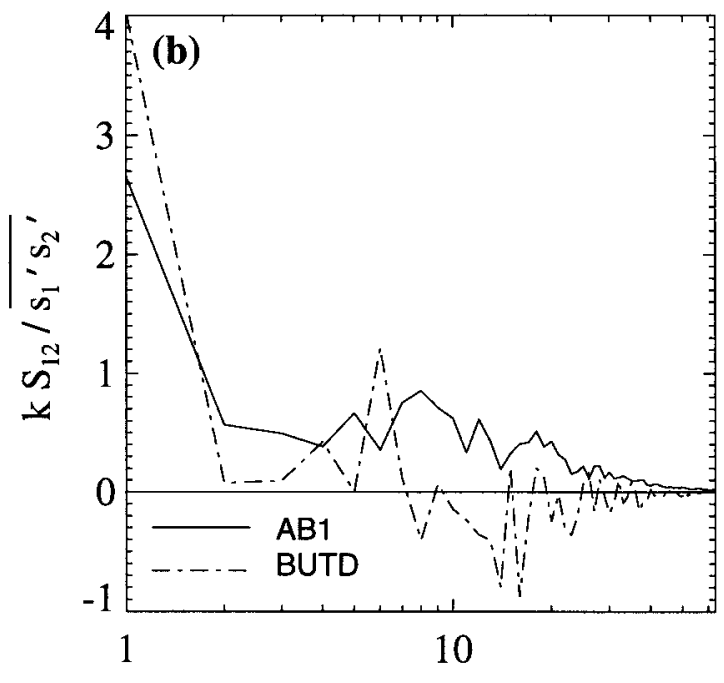

$\mathrm{k}$

FIG. B1. (a) Spectral correlations and (b) cospectra of bottom-up diffusing scalars BU or A (scalar values denoted by $s_{1}$ ) and top-down diffusing scalars TD or B (scalar values denoted by $s_{2}$ ) determined from LES. The plotted quantities have been calculated at height $z / z_{i}=$ 0.14. The normalization in (b) is chosen such that $\int_{\ln 1}^{\ln 63} k S_{12}^{\prime} d(\ln k)=1$, with $S_{12}^{\prime} \equiv S_{12} / \overline{s_{1}^{\prime} s_{2}^{\prime}}$. 


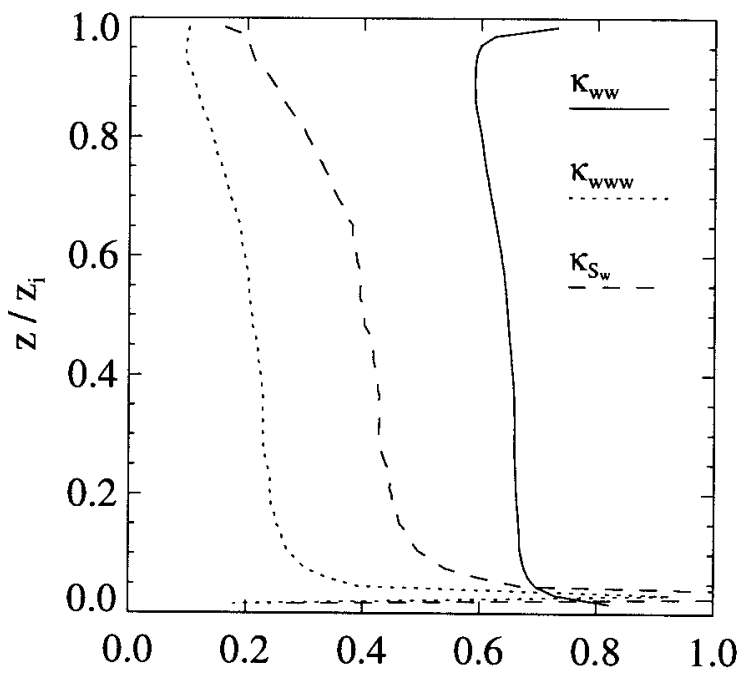

$\kappa$

FIG. C1. Ratios of top-hat contribution to total quantity for $\overline{w^{\prime 2}}$, $\overline{w^{\prime 3}}$, and $S_{w}$ determined from LES.

\section{APPENDIX C}

\section{Top-Hat Formulas for $a$ and $M$}

In order to drive a mass-flux scheme, one needs to know the updraft area fraction $a$ and the mass flux $M$. Use can be made of top-hat formulas that relate $a$ and $M$ to the turbulent vertical velocity statistics $\overline{w^{\prime 2}}$ and $\overline{w^{\prime 3}}$. In this appendix we will present these top-hat formulas and evaluate their performance, given that we already know the profiles of $\overline{w^{\prime 2}}$ and $\overline{w^{\prime 3}}$.

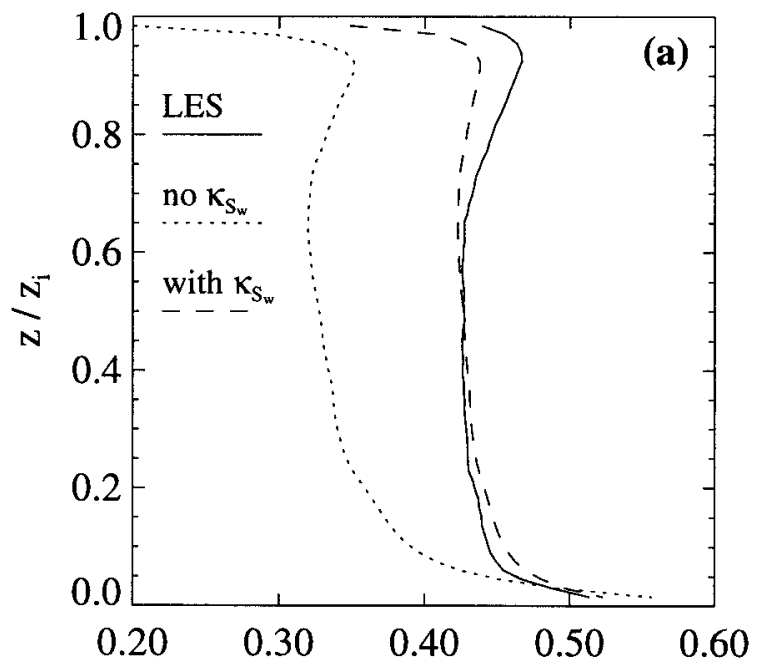

a
Using the following top-hat formulas for $a$ and $M$ from Randall et al. (1992),

$$
\begin{aligned}
a & =\frac{1}{2}-\frac{S_{w, \text { top-hat }}}{2 \sqrt{4+S_{w, \text { top-hat }}^{2}}}, \text { and } \\
M & =\frac{\left(\overline{w^{\prime 2}}\right)_{\text {top-hat }}^{1 / 2}}{\sqrt{4+S_{w, \text { top-hat }}^{2}}},
\end{aligned}
$$

where the top-hat skewness is used, defined as $S_{w, \text { top-hat }}$ $\equiv \overline{w^{\prime 3}}$ top-hat $/\left(\overline{w^{\prime 2}}\right)_{\text {top-hat }}^{3 / 2}$, and relating all top-hat quantities in (C.1) and (C.2) to the total quantities using $\kappa$-factors, we can write the following equations:

$a=\frac{1}{2}-\frac{\kappa_{s_{w}}(z) S_{w}}{2 \sqrt{4+\kappa_{s_{w}}^{2}(z) S_{w}^{2}}} \approx \frac{1}{2}-\frac{\kappa_{s_{w}} S_{w}}{4}$, and

$M=\frac{\sqrt{\kappa_{w w}(z)}\left(\overline{w^{\prime 2}}\right)^{1 / 2}}{\sqrt{4+\kappa_{s_{w}}^{2} S_{w}^{2}}} \approx \frac{\sqrt{\kappa_{w w}}\left(\overline{w^{\prime 2}}\right)^{1 / 2}}{2}$.

The $\kappa$ factors are defined as $\kappa_{w w} \equiv \overline{w^{\prime 2}}$ top-hat $\overline{/ w^{\prime 2}}$ and $\kappa_{S_{w}} \equiv S_{w, \text { top-hat }} / S_{w}$. In the approximating step of (C.3) and (C.4), it is assumed that the $\kappa$ factors are independent of height and that $\kappa_{S_{w}}^{2} S_{w}^{2} / 4 \ll 1$. In Fig. C1 we plot the profiles of the $\kappa$ factors. The top-hat fraction of the total vertical velocity variance $\kappa_{w w}$ turns out to have small variation with height (it decreases from 0.7 near the bottom to 0.6 near the top of the CBL) and on average it is close to the theoretical value for a Gaussian pdf $P\left(w^{\prime}, s^{\prime}\right)$, namely 0.64 . However, the top-hat fraction of the total third moment of the turbulent vertical velocity $\kappa_{w w w}$ is much lower (about 0.2 ) and less constant with height. Note that under Gaussian assumptions $\kappa_{w w w}$ is undefined, since in that case $\overline{w^{\prime 3}}=0$. The top-hat

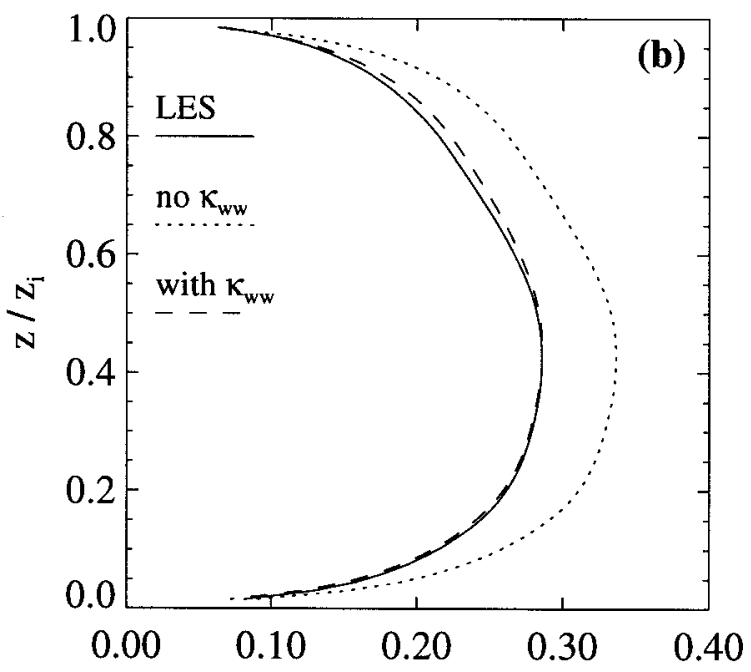

FIG. C2. (a) Updraft area fraction and (b) mass flux, determined from LES. The lines labeled "with $\kappa_{S_{w}}$ " and "with $\kappa_{w w}$ " correspond to Eqs. (C3) and (C4), respectively, and the lines labeled "no $\kappa_{S_{w}}$ " and "no $\kappa_{w w}$ " correspond to the same equations but without the $\kappa$ 's (or, equivalently, with the $\kappa$ 's put equal to 1 ). 
fraction of the total skewness $\kappa_{S_{w}}$ is found to be about 0.4 .

In Figs. C2a,b we show $a$ and $M$ calculated with and without the $\kappa$ factors and compared to LES. The LES profiles were used to drive the mass-flux schemes in this paper. Consistent with Sorbjan (1996) the LES result for $a$ shows a roughly constant value of 0.43 in the bulk of the CBL. The LES result for $M$ shows a profile that is nearly symmetrical around its maximum of $0.29 w_{*}$. We can conclude from Figs. C2a,b that (C.3) and (C.4), using $\kappa_{S_{w}}=0.4$ and $\kappa_{w w}=0.64$, give a good performance. However, if we do not use the $\kappa$ factors the minimum of $a$ becomes 0.33 and the maximum of $M$ becomes $0.34 w_{*}$. So, large errors in the estimation of $a$ and $M$ are made if one does not take the $\kappa$ factors into account.

\section{REFERENCES}

Arakawa, A., and W. H. Schubert, 1974: Interaction of a cumulus cloud ensemble with the large-scale environment, Part I. J. Atmos. Sci., 31, 674-701.

Beets, C., M. J. Molemaker, and J. Vilà-Guerau de Arellano, 1996: Direct numerical simulation and large-eddy simulation of a nonpremixed binary reaction in a turbulent convective boundary layer. Engineering Turbulence Modelling and Experiments 3, W. Rodi and G. Bergeles, Eds., Elsevier, 279-287.

Brown, A. R., 1996: Evaluation of parameterization schemes for the convective boundary layer using large-eddy simulation results. Bound.-Layer Meteor., 81, 167-200.

Businger, J. A., and S. P. Oncley, 1990: Flux measurement with conditional sampling. J. Atmos. Oceanic Technol., 7, 349-352.

Chatfield, R. B., and R. A. Brost, 1987: A two-stream model of the vertical transport of trace species in the convective boundary layer. J. Geophys. Res., 92, 13 263-13 276.

de Laat, A. T. J., and P. G. Duynkerke, 1998: Analysis of ASTEXstratocumulus observational data using a mass-flux approach. Bound.-Layer Meteor., 86, 63-87.

Fitzjarrald, D. R., and D. H. Lenschow, 1983: Mean concentration and flux profiles for chemically reactive species in the atmospheric surface layer. Atmos. Environ., 17, 2505-2512.

Galmarini, S., J. Vilà-Guerau de Arellano, and P. G. Duynkerke, 1997: Scaling the turbulent transport of chemical compounds in the surface layer under neutral and stratified conditions. Quart. J. Roy. Meteor. Soc., 123, 223-242.

Gao, W., and M. L. Wesely, 1994: Numerical modeling of the turbulent fluxes of chemically reactive trace gases in the atmospheric boundary layer. J. Appl. Meteor., 33, 835-847.

Holtslag, A. A. M., and C.-H. Moeng, 1991: Eddy diffusivity and countergradient transport in the convective atmospheric boundary layer. J. Atmos. Sci., 48, 1690-1698.

Hundsdorfer, W., B. Koren, M. van Loon, and J. G. Verwer, 1995: A positive finite-difference advection scheme. J. Comput. Phys., 117, 35-46.

Hunt, J. C. R., J. C. Kaimal, and J. E. Gaynor, 1988: Eddy structure in the convective boundary layer: New measurements and new concepts. Quart. J. Roy. Meteor. Soc., 114, 827-858.

Koren, B., 1993: A robust upwind discretization method for advection, diffusion and source terms. Numerical Methods for Advection-Diffusion Problems, C. B. Vreugdenhil and B. Koren, Eds., Notes on Numerical Fluid Mechanics 45, Vieweg, 117138.

Molemaker, M. J., and J. Vilà-Guerau de Arellano, 1998: Control of chemical reactions by convective turbulence in the boundary layer. J. Atmos. Sci., 55, 568-579.

Nieuwstadt, F. T. M., and R. A. Brost, 1986: The decay of convective turbulence. J. Atmos. Sci., 43, 532-546.

— eddy simulation of the convective boundary layer: A comparison of four computer codes. Turbulent Shear Flows 8, F. Durst, R. Friedrich, and B. E. Launder, Eds., Springer-Verlag, 532-546.

Piacsek, A. P., and G. P. Williams, 1970: Conservation properties of convection difference schemes. J. Comput. Phys., 6, 392-405.

Randall, D. A., Q. Shao, and C.-H. Moeng, 1992: A second-order bulk boundary-layer model. J. Atmos. Sci., 49, 1903-1923.

Schumann, U., 1989: Large-eddy simulation of turbulent diffusion with chemical reactions in the convective boundary layer. Atmos. Environ., 23, 1713-1727.

_ and wavy surfaces. Large Eddy Simulation of Complex Engineering and Geophysical Flows, B. Galperin and S. A. Orszag, Eds., Cambridge University Press, 399-421.

— , and C.-H. Moeng, 1991a: Plume fluxes in clear and cloudy convective boundary layers. J. Atmos. Sci., 48, 1746-1757.

- , and 1991b: Plume budgets in clear and cloudy convective boundary layers. J. Atmos. Sci., 48, 1758-1770.

Siebesma, A. P., 1997: On the mass flux approach for atmospheric convection. New Insights and Approaches to Convective Parametrization, ECMWF Workshop Proceedings, 25-57. [Available from European Centre for Medium-Range Weather Forecasts, Shinfield Park, Reading RG2 9AX, United Kingdom.]

— , and J. W. M. Cuijpers, 1995: Evaluation of parametric assumptions for shallow cumulus convection. J. Atmos. Sci., 52, $650-666$.

Sorbjan, Z., 1996: Numerical study of penetrative and "solid lid" nonpenetrative convective boundary layers. J. Atmos. Sci., 53, 101-112.

Stull, R. B., 1993: Review of non-local mixing in turbulent atmospheres: Transilient turbulence theory. Bound.-Layer Meteor., 62, 21-96.

Sykes, R. I., S. F. Parker, D. S. Henn, and W. S. Lewellen, 1994: Turbulent mixing with chemical reaction in the planetary boundary layer. J. Appl. Meteor., 33, 825-834.

Tiedtke, M., 1989: A comprehensive mass flux scheme for cumulus parameterization in large-scale models. Mon. Wea. Rev., 117, 1779-1800.

van Haren, L., and F. T. M. Nieuwstadt, 1989: The behavior of passive and buoyant plumes in a convective boundary layer, as simulated with a large-eddy model. J. Appl. Meteor., 28, 818-832.

Verver, G. H. L., H. van Dop, and A. A. M. Holtslag, 1997: Turbulent mixing of reactive gases in the convective boundary layer. Bound.-Layer Meteor., 85, 197-222.

Verwer, J. G., and D. Simpson, 1995: Explicit methods for stiff ODEs from atmospheric chemistry. Appl. Numer. Math., 18, 413-430.

Vilà-Guerau de Arellano, J., and P. G. Duynkerke, 1993: Secondorder closure study of the covariance between chemically reactive species in the surface layer. J. Atmos. Chem., 16, 145155 .

, and K. F. Zeller, 1995: Atmospheric surface layer similarity theory applied to chemically reactive species. J. Geophys. Res., 100, 1397-1408.

Wyngaard, J. C., and R. A. Brost, 1984: Top-down and bottom-up diffusion of a scalar in the convective boundary layer. J. Atmos. Sci., 41, 102-112.

—, and C.-H. Moeng, 1992: Parameterizing turbulent diffusion through the joint probability density. Bound.-Layer Meteor., 60, $1-13$.

Young, G. S., 1988a: Turbulence structure of the convective boundary layer. Part II: Phoenix 78 aircraft observations of thermals and their environment. J. Atmos. Sci., 45, 727-735.

— 1988b: Turbulence structure of the convective boundary layer. Part III: The vertical velocity budgets of thermals and their environment. J. Atmos. Sci., 45, 2039-2049. 